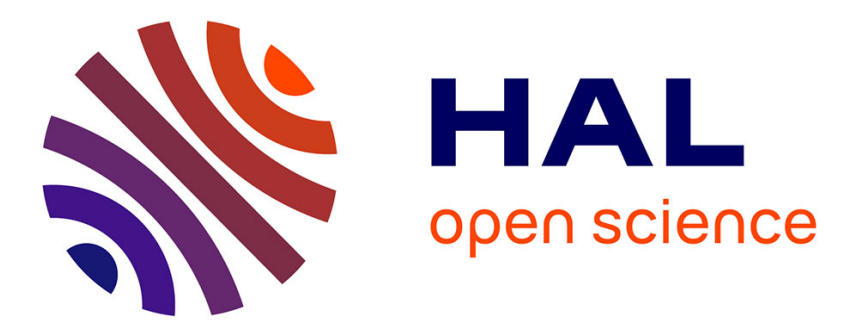

\title{
Convective and radiative thermal transfer with multiple reflections. Analysis and approximation by a finite element method
}

Jerome Monnier, Jean-Paul Vila

\section{To cite this version:}

Jerome Monnier, Jean-Paul Vila. Convective and radiative thermal transfer with multiple reflections. Analysis and approximation by a finite element method. Mathematical Models and Methods in Applied Sciences, 2001, 11 (2), pp.229-262. 10.1142/S0218202501000854 . inria-00256540

\section{HAL Id: inria-00256540 \\ https://hal.inria.fr/inria-00256540}

Submitted on 15 Feb 2008

HAL is a multi-disciplinary open access archive for the deposit and dissemination of scientific research documents, whether they are published or not. The documents may come from teaching and research institutions in France or abroad, or from public or private research centers.
L'archive ouverte pluridisciplinaire HAL, est destinée au dépôt et à la diffusion de documents scientifiques de niveau recherche, publiés ou non, émanant des établissements d'enseignement et de recherche français ou étrangers, des laboratoires publics ou privés. 


\title{
CONVECTIVE AND RADIATIVE THERMAL TRANSFER WITH MULTIPLE REFLECTIONS. ANALYSIS AND APPROXIMATION BY A FINITE ELEMENT METHOD
}

\author{
J. MONNIER \\ Laboratoire de Modélisation et Calcul (LMC-IMAG) \\ BP 53, F-38041 Grenoble Cedex 9, France. \\ J.P. VILA \\ Institut National des Sciences Appliquées de Toulouse (INSA) \\ Département Génie Mathématique UMR CNRS 5640 \\ Modélisation pour l'Industrie et la Physique, F-31077 Toulouse Cedex, France.
}

\begin{abstract}
We study a 3D steady-state thermal model taking into account heat transfer by convection, diffusion and radiation with multiple reflections (grey bodies). This model is a nonlinear integrodifferential system which we solve numerically by a finite element method. Some results of existence and uniqueness of the solution are proved, the numerical analysis is detailed, error estimates are given and twodimensional numerical results of thermal exchanges under a car bonnet are presented.

Keywords: Elliptic partial differential equations, non local boundary condition, finite element method, radiative heat transfer, grey bodies.
\end{abstract}

\section{Introduction}

This paper deals with a steady-state thermal model taking into account the three different heat exchanges: convection, diffusion and radiation. The model of radiation takes into account the emission, the reflection and the absorption of the radiant energy. The emitted and reflected radiation are diffusely distributed. The surfaces are assumed to be opaque and to behave like grey bodies, i.e. the radiative exchanges do not depend on wavelength. In other respects, we assume that the surfaces are separated by a non participating media (hence the radiative transfer is apparent in the boundary conditions). The radiative heat transfer is described by the radiosity: the radiosity being the radiant energy which flows away from a surface.

We denote by $\vec{u}$ the fluid velocity, by $\theta$ the fluid temperature and by $w$ the radiosity. The unknown in our problem is $(\ddot{\theta}, w)$ and is a solution of the integrodifferential system containing the convection-diffusion equation coupled with an integral equation on the boundary. The boundary condition of the system is non linear, non local and non monotone.

The present paper details a mathematical analysis and a numerical analysis of this thermal model. Furthermore, an algorithm to solve the problem is presented and implemented.

The mathematical analysis of this model has already been partially studied in different papers. Let us describe briefly these existing works.

Firstly, a mathematical analysis of the present radiative model without the convective term has been carried out in ${ }^{10}$ and ${ }^{11}$. The integral equation is studied in detail and the authors give an explicit expression for its solution $w$ which has permitted them to study the partial differential equation with only the temperature 
$\theta$ as an unknown. Then, they truncate the non linearity and prove the existence of a minimal and a maximal solution to the truncated problem. This technique of truncation has permitted them notably to write the analysis in the standard Sobolev space $H^{1}(\Omega)$.

Our paper ${ }^{2}$ treated a shape optimal design problem where the direct problem contained the present thermal model. In our last paper, we wrote a mathematical analysis of the thermal model used here employing the idea of truncation from 11. We proved that the truncated problem has a solution in $H^{1}(\Omega)$ and by stating a weak maximum principle, we obtained the existence of a solution to the initial problem (the proofs presented therein are not all detailed). We note that in both references ${ }^{11}$ and ${ }^{2}$ some proof sketches of uniqueness are presented but none are complete. Also, we presented in ${ }^{2}$, an outline of the numerical analysis.

This paper completes ${ }^{2}$ : firstly, we detail the missing proofs of the mathematical analysis and then we prove some extra results; secondly, we describe in detail the numerical analysis.

In ${ }^{13},{ }^{14}$ and ${ }^{15}$ the author studies the same thermal model with a different approach. Firstly, the coupling between the boundary condition of the partial differential equation and the integral equation is treated differently and the formulation obtained takes into account the limit case of black bodies. Secondly, the author does not use the previous technique of truncation, hence the mathematical analysis is written in the Banach space $H^{1}(\Omega) \cap L^{5}(\partial \Omega)$. The author shows that if there exists a lower and an upper solution to the problem then there exists a solution in $H^{1}(\Omega) \cap L^{5}(\partial \Omega),{ }^{13} 14$. Some others results are presented (such as the maximum principle and the uniqueness of the solution), however they are incomplete. In ${ }^{15}$, the author presents a finite element error analysis of a quite different formulation of the radiative terms and there he uses an inverse monotone method.

Let us point out that in the present paper we use the technique of truncation of ${ }^{11}$ since it presents the following advantages which we now describe. Firstly, it permits us to write the mathematical analysis in the standard Sobolev space $H^{1}(\Omega)$. To this end, we prove the weak maximum principle and we obtain the existence and uniqueness of the "physical" solution (i.e. the solution which satisfies this maximum principle). Secondly, the framework with which we use to write the numerical analysis (see Theorem 3) requires (notably) the two following properties: i) the equations must be formulated as a fixed point problem $(F(x)=(I+T \circ G)(x)=0, x=(\theta, w))$; ii) the linearized problem must be well posed. Again, the truncation allows us to satisfy these properties in the Sobolev space $H^{1}(\Omega)$. This truncation thus permits us to write the mathematical analysis and the numerical analysis in the same framework.

The paper is organized as follows. In the next section, we write the equations of the physical model and the boundary value problem. In Section 3, we expound the mathematical analysis of the equations. We do not consider special restrictions on the domain $\Omega$ : it has to be a lipschitz bounded open set in $\mathbb{R}^{n}, n=2$ or 3 . With respect to the velocity $\vec{u}$, we assume that the fluid is incompressible $(\operatorname{div}(\vec{u})=0)$ and $\vec{u}$ belongs to $\left(L^{p}(\Omega)\right)^{n}$ with $p>n$. In this section, we recall some existing results in order to prove some additional results. Firstly, we solve the integral equation and we give an analytical expression of the radiosity $w$ as a function of $\theta$ (Proposition 1). Following, we obtain a partial differential equation with $\theta$ as the only unknown, the boundary condition being non-linear, non-monotone and non-local (see Section 3.2, Problem $\left(P^{Q}\right)$ ). We define the truncated problem (Problem $\left(P^{\bar{Q}}\right)$ ), and this is done by truncating the non-linear boundary conditions to "natural" values. 
This truncated problem has a solution in $H^{1}(\Omega)$ (Proposition 3). By proving that any solution of this problem satisfies the weak maximum principle (Proposition 2) it then follows straightforwardly that the initial problem has a solution $(\theta, w)$ in $H^{1}(\Omega) \times L^{\infty}(\partial \Omega)$ which satisfies the weak maximum principle (Theorem 1).

Next, we study the linearized problem and prove that if the thermal conductivity of the fluid $\lambda$ is sufficiently large compared to the radiative terms, then the linearized problem is coercitive in $H^{1}(\Omega)$. It follows from the Lax-Milgram theorem that this is well posed (Proposition 4). The study of this linearized problem is useful to the analysis of finite element schemes (Section 4) and to the analysis of shape optimal design problems where the direct problem contains the present model (see ${ }^{2},{ }^{3}$ ). Furthermore and following an idea of ${ }^{14}$, we prove that when the linearized problem is well posed, the physical solution of the (non-linear) problem is unique (Proposition 5).

In Section 4, we write the numerical analysis. The domain $\Omega$ is a subset of $\mathbb{R}^{n}$ ( $n=2$ or 3,) and it is supposed polyhedric. Using a standard finite element method, we discretize the equations of a new truncated problem. Under the assumption that the linearized problem is well posed (here, when the thermal conductivity $\lambda$ is sufficiently large) we prove the existence and uniqueness of the discrete solution and its convergence towards the unique physical solution of the initial problem when the mesh size $h$ is sufficiently small. To this end, we prove that the equations fit into the framework of ${ }^{1}$. This previous paper deals with a finite dimensional approximation of nonlinear problems of the form $F(\lambda ; x)=0$ where $F: \Lambda \times X \rightarrow X, \Lambda$ is an interval of $\mathbb{R}$ and $X$ is a Banach space. We consider branches of non-singular solutions to the problem and we use results of ${ }^{1}$. To this end, we write the continuous and discrete thermal problems as fixed point problems (Section 4.2) and we obtain branches of non-singular solutions when $\lambda$ is large enough (Section 4.3). We prove the convergence of the discrete solution of a linear and decoupled system. The error estimates are written in detail and are based on estimates of the error of projection when the function belongs to fractional order spaces (Section 4.4). Finally, we can apply results of ${ }^{1}$ (see also ${ }^{5}$ ) and obtain existence and uniqueness of the discrete solution $\left(\theta_{h}, w_{h}\right)$ and its convergence (Theorem 2). Let us point out that due to the results of ${ }^{1}$, this analysis does not require the discrete maximum principle.

In Section 5, we present the algorithm we use in order to numerically solve the problem. We consider the equations in their coupled form, i.e. the coupled partial differential equation-integral system. We linearize the nonlinear boundary condition using the Newton-Raphson algorithm and we treat the coupling using a relaxation algorithm. We introduce streamline diffusion into the finite element scheme whereby the computation of the angle factors are done using a Monte-Carlo method and shadows effects are taken into account. We then present the linear systems to be solved.

In Section 6, we consider a twodimensional and stationary incompressible potential flow under a car bonnet and we present numerical results of heat transfer modeled by the equations studied previously.

\section{Physical Model}

We consider a stationary incompressible fluid flow and we seek to model heat transfer by convection, diffusion and radiation. For the radiation model, we consider multiple reflection effects i.e. we take into account the emission, reflection and absorption. We assume that the surfaces are opaque and grey, hence the radiative exchanges do not depend on the wave length. In addition, the surfaces are separated by a radiatively non participating media and the emitted and reflected radiation are diffusely distributed (see e.g. ${ }^{12}$ ). Under these assumptions, the radiative transfer 
are described by the radiosity, it is the radiant energy which flows away from a surface per unit area, it is denoted by $w$.

We denote by $\theta$ the fluid temperature and by $\vec{u}$ its velocity. Let $\Omega$ be a Lipschitz bounded open set in $\mathbb{R}^{n}$ ( $n=2$ or 3$)$ and $\partial \Omega$ its boundary. The radiosity $w$ is the solution of the following Fredholm integral equation of the second kind called the radiosity equation (see e.g. ${ }^{12}$ ):

$$
w(x)=(1-\varepsilon(x)) \int_{\partial \Omega} \phi(x, y) w(y) d s(y)+\varepsilon(x) \sigma \theta^{4}(x) \quad \text { on } \partial \Omega
$$

where $\sigma$ is the Stephan-Boltzmann's constant. The function $\varepsilon$ is the surface emittance and satisfies:

$$
0<\varepsilon_{0} \leq \varepsilon(x) \leq \varepsilon_{1}<1 \text { on } \partial \Omega
$$

The kernel $\phi \in L^{1}(\partial \Omega \times \partial \Omega)$ is the angle factor, it is positive, symmetric and satisfies:

$$
\int_{\partial \Omega} \phi(x, y) d s(x)=1
$$

We refer for instance to ${ }^{12}$ for the expression of the angle factor $\phi(x, y)$. The conservation of energy gives:

$$
-\lambda \Delta \theta+\rho C_{p} \vec{u} \cdot \vec{\nabla} \theta=0 \text { in } \Omega
$$

where $\lambda, \rho$ and $C_{p}$ are respectively the thermal conductivity, the density and the specific heat (at constant pressure) of the fluid.

We consider on a part of the boundary denoted by $\Gamma_{f}$, heat transfer by radiation and convection. If we denote by $h$ the thermal transfer coefficient and by $\theta_{0}$ the outside temperature, it follows from Fourier's law that:

$$
-\lambda \vec{\nabla} \theta \cdot \vec{n}=h\left(\theta-\theta_{0}\right)+\frac{\varepsilon}{1-\varepsilon}\left(\sigma \theta^{4}-w\right) \quad \text { on } \Gamma_{f}
$$

where $w$ is solution of (2.1) and $n$ is the external normal to $\partial \Omega$. For a sake of simplicity, we assume that the thermal transfer coefficient $h$ does not depend on the fluid velocity $\vec{u}$, it is a constant. We denote by $\Gamma_{d}$ the part of the boundary where the temperature is known (Dirichlet's condition) and we denote by $\Gamma_{n}$ the part of the boundary where heat exchanges are null (Neumann's condition). We have $\Gamma_{d} \cup \Gamma_{n} \cup \Gamma_{f}=\partial \Omega$ and $\operatorname{meas}\left(\Gamma_{d}\right)>0$.

The fluid velocity $\vec{u}$ is given and we make the following assumptions: Assumption 1 The fluid is incompressible (div $(\vec{u})=0$ in $\Omega$ ) and there exists a real $p>n$ such that $\vec{u} \in\left(L^{p}(\Omega)\right)^{n}$.

Notice that under Assumption 1, $\vec{u}$ belongs to $L^{2}(\Omega)^{n}$ and is divergence free, hence $\vec{u}$ has a normal trace in $H^{-\frac{1}{2}}(\partial \Omega)$. On the other hand, we do not require that $\vec{u}$ belongs to $\left(L^{\infty}(\Omega)\right)^{n}$.

Assumption 2 The boundary parts denoted by $\Gamma_{n}$ and $\Gamma_{f}$ are such that $\vec{u} \cdot \vec{n}$ is positive on $\Gamma_{n} \cup \Gamma_{f}$.

Dimensionless model We denote by $P e$ the Peclet number, $P e=\frac{\rho C_{p} U^{*} L^{*}}{\lambda}$, where $U^{*}$ is a characteristic velocity of the flow and $L^{*}$ is a characteristic length. We denote the Biot number $B i, B i=\frac{h L^{*}}{\lambda}$ ( $B i$ is constant), and the dimensionless reals $\delta_{1}=\frac{L^{*} \sigma\left(\theta^{*}\right)^{3}}{\lambda}$ and $\delta_{2}=\frac{L^{*} w^{*}}{\lambda \theta^{*}}$, where $\theta^{*}$ is a characteristic temperature and $w^{*}$ 
is a characteristic radiative energy,

The dimensionless model is, see e.g. ${ }^{12}$ :

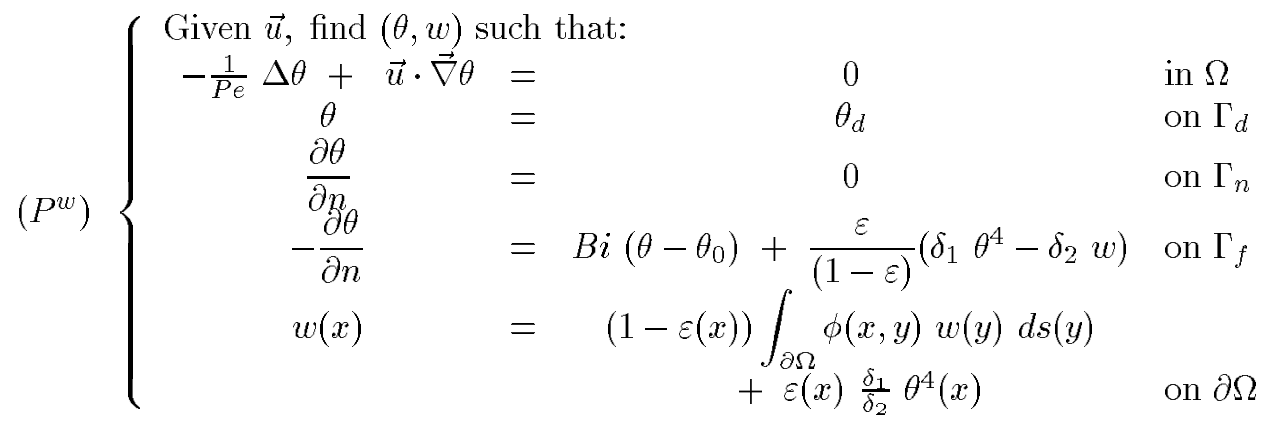

In the remainder of the paper, $\vec{u}, \theta$ and $w$ denote the dimensionless variables and we omit the arrow above the vectorial entities.

Remark 1 The formulation presented above do not consider the case of black bodies (i.e. $\varepsilon=1$ ), contrary to the one studied in ${ }^{14}, 9$. In such a case, for example when $\varepsilon(x)=1$ on $\Gamma_{b}, \Gamma_{b} \subset \partial \Omega$ and $0<\varepsilon(x)<1$ on $\Gamma_{d} \cup \Gamma_{n} \cup \Gamma_{f}$ with $\Gamma_{d} \cup \Gamma_{n} \cup \Gamma_{f} \cup \Gamma_{b}=$ $\partial \Omega$, we could introduce the following extra boundary condition in $\left(P^{w}\right)$ :

$$
-\frac{\partial \theta}{\partial n}=B i\left(\theta-\theta_{0}\right)+\left[\delta_{1} \theta^{4}-\delta_{2} \int_{\partial \Omega} \phi(x, y) w(y) d s(y)\right] \text { on } \Gamma_{b}
$$

\section{Mathematical analysis}

Let us recall that some results of existence and uniqueness related to this radiative model (with or without the convective term) have been proved by different authors. Briefly, in ${ }^{10}$ and ${ }^{11}$, the existence of a solution to a truncated problem is obtained, using either a lower and upper solution method or the Schauder fixed point theorem. $\operatorname{In}^{8}$ and ${ }^{2}$ and following the previous idea of truncation, the existence of a solution to the present model which satisfies the maximum principle is stated using the Schauder fixed point theorem either on the coupled formulation as $\left(P^{w}\right)$ or on the formulation $\left(P^{\bar{Q}}\right)$ below. In these previous references and thanks to the technique of truncation, the analysis is written in the standard Sobolev space $H^{1}(\Omega)$. On the other hand, in ${ }^{13}$ and ${ }^{14}$, the author does not use this technique. He proves that if there exists a lower and an upper solution, then there exists a solution in $H^{1}(\Omega) \cap L^{5}(\partial \Omega)$.

The present section completes the analysis presented in ${ }^{2}$. To this end, we use the analysis of the integral equation done in ${ }^{11}$ and we follow the idea of truncation. We define the truncated problem and we recall the existence of a solution in $H^{1}(\Omega)$ (Proposition 3). We prove that any solution of this truncated problem satisfies the weak maximum principle (Proposition 2) and we obtain the existence of a physical solution to the initial problem i.e. a solution which satisfies the maximum principle (Theorem 1). Next, we study the linearized problem and obtain that if the thermal conductivity $\lambda$ is sufficiently large compared to the radiative terms then the linearized problem is coercitive in $H^{1}(\Omega)$, hence well posed (Proposition 4). Following an idea of ${ }^{14}$, we obtain the uniqueness of the physical solution when the linearized problem is well posed (hence when $\lambda$ is sufficiently large) (Proposition 5). 


\subsection{Step 1. Resolution of the Integral Equation}

We define the operator $A$ by:

$$
A w(x)=(1-\varepsilon(x)) \int_{\partial \Omega} \phi(x, y) w(y) d s(y)
$$

We write

$$
e=\varepsilon \frac{\delta_{1}}{\delta_{2}} \theta^{4}
$$

and we consider the following problem:

$$
\left\{\begin{array}{l}
\text { Given } e \in L^{p}(\partial \Omega), 1 \leq p \leq \infty \text {, find } w \in L^{p}(\partial \Omega) \text { such that: } \\
(I d-A) w=e
\end{array}\right.
$$

The operator $A$ is a contracting operator in $L^{p}(\partial \Omega)$ for all $p \in[1, \infty]$ hence there exists a unique solution $w$ to (3.6), see e.g. ${ }^{11}$. Moreover, let us recall the following result which is fundamental in the remainder of the analysis:

Proposition 1 (Perret and Witomski, 11). Problem (3.6) has a unique solution $w \in L^{p}(\partial \Omega)$. This solution can be written:

$$
w(x)=\int_{\partial \Omega} K(x, y) e(y) d s(y)+e(x)
$$

where the kernel $K$ belongs to $L^{1}(\partial \Omega \times \partial \Omega)$, is positive and satisfies:

$$
\begin{gathered}
\int_{\partial \Omega} K(x, y) \varepsilon(y) d s(y)=1-\varepsilon(x) \\
\int_{\partial \Omega} K(x, y) \frac{\varepsilon(x)}{1-\varepsilon(x)} d s(x)=1
\end{gathered}
$$

\subsection{Step 2. Analysis of the Partial Differential Equation}

We deduce from the analytic expression (3.7) of $w$, that Problem $\left(P^{w}\right)$ can be formulated as follows:

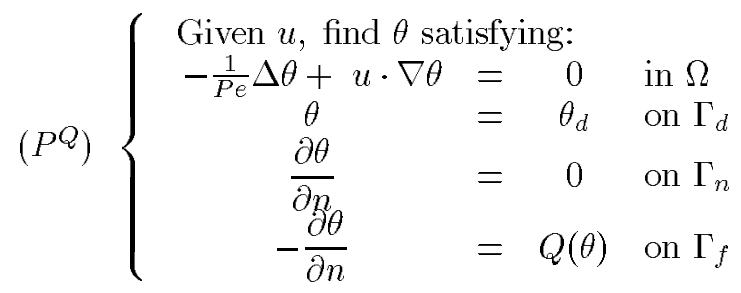

where the operator $Q$ is defined by:

$Q(\theta)(x)=B i\left(\theta-\theta_{0}\right)(x)+\left[\varepsilon(x) \delta_{1} \theta^{4}(x)-\frac{\varepsilon(x)}{1-\varepsilon(x)} \int_{\partial \Omega} K(x, y) \varepsilon(y) \delta_{1} \theta^{4}(y) d s(y)\right]$

for almost every $x$ in $\partial \Omega$.

We assume that the given temperatures $\theta_{d}$ and $\theta_{0}$ are positive functions respectively in $H^{\frac{3}{2}}\left(\Gamma_{d}\right)$ and $H^{\frac{1}{2}}\left(\Gamma_{f}\right) \cap L^{\infty}\left(\Gamma_{f}\right)$.

The basic idea of the following analysis is the truncation of the non linear, non local operator $Q$. This idea is due to ${ }^{11}$. 
The truncated problem We define:

$$
\theta_{i n f}=\operatorname{Min}\left(\inf _{\Gamma_{d}} \theta_{d}, \inf _{\Gamma_{f}} \theta_{0}\right) ; \theta_{\text {sup }}=\operatorname{Max}\left(\sup _{\Gamma_{d}} \theta_{d}, \sup _{\Gamma_{f}} \theta_{0}\right)
$$

and

$$
\bar{\theta}(x)=\left\{\begin{array}{clc}
\theta_{\text {inf }} & \text { if } & \theta(x) \leq \theta_{\text {inf }} \text { a.e } \\
\theta(x) & \text { if } & \theta_{\text {inf }} \leq \theta(x) \leq \theta_{\text {sup }} \\
\theta_{\text {sup }} & \text { if } & \theta(x) \geq \theta_{\text {sup }} \text { a.e }
\end{array}\right.
$$

The truncated operator denoted by $\bar{Q}$ is defined as follows:

$$
\begin{aligned}
\bar{Q}(\theta)(x)= & B i\left(\bar{\theta}-\theta_{0}\right)(x)+\left[\varepsilon(x) \delta_{1}(\bar{\theta})^{4}(x)\right. \\
& \left.-\frac{\varepsilon(x)}{1-\varepsilon(x)} \int_{\partial \Omega} K(x, y) \varepsilon(y) \delta_{1}(\bar{\theta})^{4}(y) d s(y)\right]
\end{aligned}
$$

for almost every $x$ on $\partial \Omega$. And the truncated problem is the following:

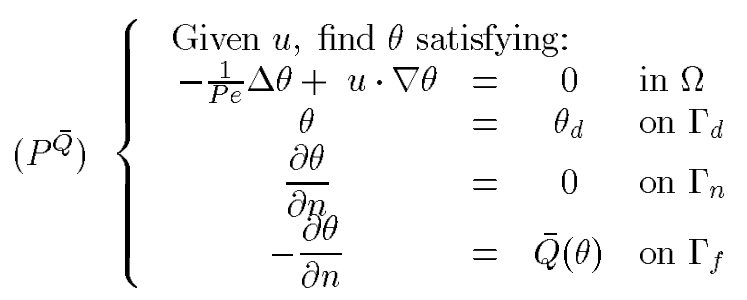

Let us recall some properties of the truncated operator $\bar{Q}$. These properties are of great importance in the remainder of the paper.

Lemma $1\left({ }^{10},{ }^{11}\right)$. The operator $\bar{Q}$ satisfies the three following properties:

i) It is a Lipschitz operator from $L^{2}(\partial \Omega)$ into $L^{2}(\partial \Omega)$. More precisely, for all $\theta_{1}, \theta_{2}$ in $L^{2}(\partial \Omega)$

$$
\left\|\bar{Q}\left(\theta_{1}\right)-\bar{Q}\left(\theta_{2}\right)\right\|_{0, \partial \Omega} \leq K\left\|\theta_{1}-\theta_{2}\right\|_{0, \partial \Omega}
$$

with

$$
K=\left[B i+4 \varepsilon_{1} \delta_{1} \theta_{\text {sup }}^{3}\left(1+\frac{1-\varepsilon_{0}}{1-\varepsilon_{1}} \sqrt{\frac{\varepsilon_{1}}{\varepsilon_{0}}}\right)\right]
$$

ii) The range of $L^{2}(\partial \Omega)$ by $\bar{Q}$ is bounded into $L^{\infty}(\partial \Omega)$. More precisely,

$$
\forall t \in L^{2}(\partial \Omega), \quad|\bar{Q}(\theta)(x)| \leq M \text { a.e. on } \partial \Omega
$$

with

$$
M=B_{i}\left(\theta_{\text {sup }}-\theta_{\text {inf }}\right)+2 \delta_{1} \varepsilon_{1} \theta_{\text {sup }}^{4}
$$

iii) If $\theta(x) \geq \theta_{\text {sup }}\left(\right.$ resp. $\left.\theta(x) \leq \theta_{\text {inf }}\right)$ then $\bar{Q}(\theta)(x) \geq 0($ resp. $\bar{Q}(\theta)(x) \leq 0)$.

The proof is done in ${ }^{10},{ }^{11}$ (see also ${ }^{8}$ ). 
The weak maximum principle We state below a weak maximum principle for the truncated problem. This maximum principle will permit us to obtain the existence of a solution to the initial problem. This result has been presented in ${ }^{2}$ but the proof has not been detailed.

Proposition 2 Let the assumptions 1 and 2 be satisfied. Any solution $\theta$ in $H^{1}(\Omega)$ of Problem $\left(P^{\bar{Q}}\right)$ satisfies:

$$
\theta_{\text {inf }} \leq \theta \leq \theta_{\text {sup }} \text { a.e. in } \Omega \cup \Gamma_{n} \cup \Gamma_{f}
$$

Proof. We define the following spaces:

$$
V_{0}(\Omega)=\left\{\theta \in H^{1}(\Omega) ;\left.\theta\right|_{\Gamma_{d}}=0\right\} \text { and } V_{t}(\Omega)=\left\{\theta \in H^{1}(\Omega) ;\left.\theta\right|_{\Gamma_{d}}=\theta_{d}\right\}
$$

Let $p>n$, we define the trilinear mapping $a:\left(L^{p}(\Omega)\right)^{n} \times V_{0}(\Omega) \times V_{0}(\Omega) \rightarrow \mathbb{R}$ such that:

$$
a(u ; \theta, t)=\frac{1}{P e} \int_{\Omega} \nabla \theta \nabla t d x+\int_{\Omega} u \nabla \theta t d x
$$

The weak formulation of $\left(P^{\bar{Q}}\right)$ is:

$$
\left\{\begin{array}{l}
\text { Find } \theta \in V_{t}(\Omega) \text { such that : } \\
\forall t \in V_{0}(\Omega), a(u ; \theta, t)+\frac{1}{P e} \int_{\Gamma_{f}} \bar{Q}(\theta) t d s=0
\end{array}\right.
$$

We choose $t=\left(\theta-\theta_{\text {sup }}\right)^{+}$as test function, where $\theta^{+}=\operatorname{Max}(\theta, 0)$. We have $t$ in $V_{0}(\Omega)$ and

$$
\nabla t=\mid \begin{array}{ll}
\nabla \theta & \text { if } \quad \theta>\theta_{\text {sup }} \\
0 & \text { if } \quad \theta \leq 0
\end{array}
$$

We obtain:

$$
\frac{1}{P e} \int_{\Omega}|\nabla t|^{2} d x+\int_{\Omega} u \nabla t t d x+\frac{1}{P e} \int_{\Gamma_{f}} \bar{Q}(t) t d s=0
$$

It follows from Lemma 1 iii) that:

$$
\int_{\Gamma_{f}} \bar{Q}(t) t d s \geq 0
$$

Furthermore, we have:

$$
\int_{\Omega} u \nabla t t d x \geq 0
$$

As a matter of fact, for all $\varphi \in \mathcal{D}_{0}(\Omega)=\left\{\varphi \in \mathcal{C}^{\infty}(\bar{\Omega}), \varphi=0\right.$ on $\left.\Gamma_{d}\right\}$, we have:

$$
\begin{aligned}
\int_{\Omega} u \nabla \varphi \varphi d x & =\int_{\Omega} u \nabla\left(\frac{\varphi^{2}}{2}\right) d x \\
& =\int_{\Omega}\left(\operatorname{div}\left(u \frac{\varphi^{2}}{2}\right)-\frac{\varphi^{2}}{2} \operatorname{div} u\right) d x
\end{aligned}
$$


Using the relation $\operatorname{div}(u)=0$ and Assumption 2, we obtain:

$$
\int_{\Omega} u \nabla \varphi \varphi d x=\left\langle u . n, \frac{\varphi^{2}}{2}\right\rangle_{H^{-\frac{1}{2}} \times H^{\frac{1}{2}}\left(\Gamma_{f} \cup \Gamma_{n}\right)} \geq 0
$$

Then, (3.22) follows from an argument of density.

We deduce from (3.20)-(3.22) that $\|\nabla t\|_{L^{2}(\Omega)}^{2} \leq 0$. It follows from the generalized Poincaré inequality that $t=0$ a.e. in $\Omega$, hence $\theta \leq \theta_{\text {sup }}$ a.e. in $\Omega$. Then the inequality $\theta \leq \theta_{\text {sup }}$ a.e. on $\partial \Omega$ is established using the continuity of the trace mapping.

The inequality $\theta \geq \theta_{\text {inf } f}$ is obtained using similar arguments with $t=\left(\theta-\theta_{\text {inf }}\right)^{-}$ with $t^{-}=\max (-t, 0)$.

Existence of solutions We have the following result of existence related to the truncated problem.

Proposition $3\left({ }^{2},{ }^{2}\right)$. Under the assumptions 1 and 2 , the truncated problem $\left(P^{\bar{Q}}\right)$ has a solution in $H^{1}(\Omega)$.

Without the convective term, the proof is done in ${ }^{11}$ using a method of lower and upper solutions. More precisely, it is proved that $\left(P^{\bar{Q}}\right)$ has a minimal and a maximal solution in $H^{1}(\Omega)$. This last proof can be extended to the case $u \neq 0$.

With the convective term, the proof is done in ${ }^{2},{ }^{8}$ using the Schauder fixed point theorem.

Using the definition of the truncated operator, and using the propositions 2, 3, 1 , we obtain straightforwardly the existence of a solution to the initial problem.

Theorem 1 Under the assumptions 1 and 2 , there exists a solution $(\theta, w) \in H^{1}(\Omega) \times$ $L^{\infty}(\partial \Omega)$ to Problem $\left(P^{w}\right)$ which satisfies (3.16).

In others words, we proved that Problem $\left(P^{w}\right)$ has at least one physical solution.

The linearized problem Now, we study the linearized problem. This study is useful to the finite element analysis in next section as well as to the analysis of a shape optimal design problem where the direct problem contains the present model, 23 . Furthermore, it provides a proof of uniqueness of the solution (Proposition 5).

Let $\tilde{\theta}$ be a function of $H^{1}(\Omega)$ which satisfies (3.16), we define the linear operator $L: L^{2}(\partial \Omega) \rightarrow L^{2}(\partial \Omega)$ by:

$$
\begin{aligned}
L(\eta)(x)= & B i \eta(x)+4 \delta_{1}\left[\varepsilon(x)(\tilde{\theta})^{3}(x) \eta(x)\right. \\
& \left.-\frac{\varepsilon(x)}{1-\varepsilon(x)} \int_{\partial \Omega} K(x, y) \varepsilon(y)(\tilde{\theta})^{3}(y) \eta(y) d s(y)\right] \text { a.e. on } \partial \Omega(3.23)
\end{aligned}
$$

and we consider the following linearized problem:

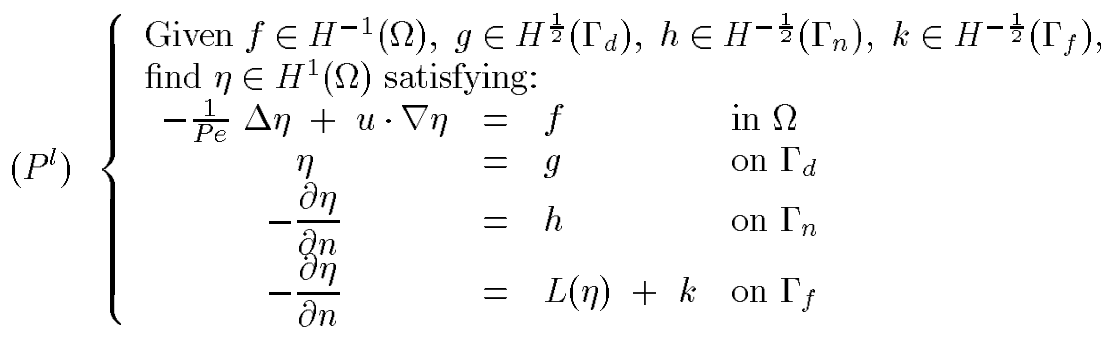


We define the Poincaré's constant by

$$
C_{\Omega}=\sup _{\eta \in V_{0}(\Omega)} \frac{\|\eta\|_{0, \Omega}}{\|\nabla \eta\|_{0, \Omega}}
$$

and

$$
\lambda_{\min }=4\left(C_{\Omega}^{2}+1\right) \varepsilon_{1} \sigma \theta_{\text {sup }}^{3}\left(\theta^{*}\right)^{3} L^{*}
$$

Proposition 4 If the thermal conductivity $\lambda$ is strictly greater than $\lambda_{\min }$ then the linearized problem $\left(P^{l}\right)$ is well posed.

Proof. The proof is done using the Lax-Milgram theorem. Let us define the trilinear mapping $c:\left(L^{p}(\Omega)\right)^{n} \times V_{0}(\Omega) \times V_{0}(\Omega) \rightarrow \mathbb{R}$ such that:

$$
c(u ; \eta, t)=a(u ; \eta, t)+\frac{1}{P e} \int_{\Gamma_{f}}\left(B i+4 \varepsilon \delta_{1} \tilde{\theta}^{3}\right) \eta t d s
$$

where the mapping $a$ is defined by (3.18). We define the trilinear mapping $l$ : $\left(L^{p}(\Omega)\right)^{n} \times V_{0}(\Omega) \times V_{0}(\Omega) \rightarrow \mathbb{R}$ by:

$$
\begin{aligned}
l(u ; \eta, t) & =a(u ; \eta, t)+\frac{1}{P e} \int_{\Gamma_{f}} L(\eta) t d s \\
& =c(u ; \eta, t)-I(\eta, t)
\end{aligned}
$$

with

$$
I(\eta, t)=\frac{4}{P e} \int_{\Gamma_{f}} \frac{\varepsilon(x)}{1-\varepsilon(x)} \int_{\partial \Omega} K(x, y) \varepsilon(y) \delta_{1} \tilde{\theta}^{3}(y) \eta(y) d s(y) t(x) d s(x)
$$

As usual, we denote by $V_{t}(\Omega)$ the translated space of $V_{0}(\Omega)$ and we write the weak formulation of $\left(P^{l}\right)$ :

$\left\{\begin{array}{l}\text { Find } \eta \in V_{t}(\Omega) \text { such that: } \forall t \in V_{0}(\Omega), \\ l(u ; \eta, t)=\langle f, t\rangle_{H^{-1} \times H^{1}(\Omega)}-\frac{1}{P e}\left[\langle h, t\rangle_{H^{-\frac{1}{2}} \times H^{\frac{1}{2}}\left(\Gamma_{n}\right)}+\langle k, t\rangle_{H^{-\frac{1}{2}} \times H^{\frac{1}{2}}\left(\Gamma_{f}\right)}\right]\end{array}\right.$

We write below a condition such that the bilinear form $l(u ; .,$.$) is V_{0}$-elliptic. Under Assumption 1 and using an argument of density, we obtain:

$$
\forall \eta \in V_{0}(\Omega), a(u ; \eta, \eta) \geq \frac{1}{P e}\|\nabla \eta\|_{0, \Omega}^{2}
$$

And, since $\tilde{\theta}$ satisfies (3.16), we have:

$$
\forall \eta \in V_{0}(\Omega), c(u ; \eta, \eta) \geq \frac{1}{P e}\|\nabla \eta\|_{0, \Omega}^{2}+\frac{1}{P e}\left[4 \varepsilon_{0} \delta_{1} \theta_{i n f}^{3}+B i\right]\|\eta\|_{0, \Gamma_{f}}^{2}
$$

Using the generalized Poincaré's inequality we have:

$$
c(u ; \eta, \eta) \geq \frac{1}{P e\left(C_{\Omega}^{2}+1\right)}\|\eta\|_{1, \Omega}^{2}+\frac{1}{P e}\left[4 \varepsilon_{0} \delta_{1} \theta_{i n f}^{3}+B i\right]\|\eta\|_{0, \Gamma_{f}}^{2}
$$


where $C_{\Omega}$ is defined by $(3.24)$.

Now, we bound above the term $I(\eta, \eta)$ in the expression of $l(u ; \eta, \eta)$. We deduce from Fubini's theorem and (3.16) that: $\forall \eta \in V_{0}(\Omega)$,

$$
\begin{aligned}
|I(\eta, \eta)| & \leq \frac{4}{P e} \delta_{1} \theta_{\text {sup }}^{3}\left|\int_{\Gamma_{f}} \int_{\partial \Omega} \frac{\varepsilon(x)}{1-\varepsilon(x)} K(x, y) \varepsilon(y) \eta(y) \eta(x) d s(y) d s(x)\right| \\
& \leq \frac{2}{P e} \delta_{1} \theta_{\text {sup }}^{3} \int_{\Gamma_{f}} \int_{\partial \Omega} \frac{\varepsilon(x)}{1-\varepsilon(x)} K(x, y) \varepsilon(y)\left[\eta^{2}(y)+\eta^{2}(x)\right] d s(y) d s(x)
\end{aligned}
$$

It follows from (3.8) and (3.9) that: $\forall \eta \in V_{0}(\Omega)$,

$$
\begin{aligned}
|I(\eta, \eta)| & \leq \frac{2}{P e} \delta_{1} \theta_{\text {sup }}^{3}\left[\int_{\partial \Omega} \varepsilon(y) \eta^{2}(y) d s(y)+\int_{\Gamma_{f}} \varepsilon(x) \eta^{2}(x) d s(x)\right] \\
& \leq \frac{4}{P e} \varepsilon_{1} \delta_{1} \theta_{\text {sup }}^{3}\|\eta\|_{0, \partial \Omega}^{2}
\end{aligned}
$$

And, we deduce from (3.27) and (3.28),

$$
\begin{aligned}
l(u ; \eta, \eta) & \geq \frac{1}{P e\left(C_{\Omega}^{2}+1\right)}\|\eta\|_{1, \Omega}^{2}+\frac{1}{P e}\left[4 \varepsilon_{0} \delta_{1} \theta_{i n f}^{3}+B i\right]\|\eta\|_{0, \Gamma_{f}}^{2} \\
& -\frac{4}{P e} \varepsilon_{1} \delta_{1} \theta_{\text {sup }}^{3}\|\eta\|_{0, \partial \Omega}^{2}
\end{aligned}
$$

We consider the norm of $H^{\frac{1}{2}}(\partial \Omega)$ defined by: $\|\beta\|_{\frac{1}{2}, \partial \Omega}=\inf _{\eta \in H^{1}(\Omega) / \text { trace }(\eta)=\beta}\|\eta\|_{1, \Omega}$. Hence,

$$
\forall \eta \in V_{0}(\Omega), \quad\|\eta\|_{0, \partial \Omega}^{2} \leq\|\eta\|_{\frac{1}{2}, \partial \Omega}^{2} \leq\|\eta\|_{1, \Omega}^{2}
$$

And, inequality (3.29) can be formulated: $\forall \eta \in V_{0}(\Omega)$,

$$
\begin{aligned}
l(u ; \eta, \eta) & \geq\left[\frac{1}{P e\left(C_{\Omega}^{2}+1\right)}-\frac{4}{P e} \varepsilon_{1} \delta_{1} \theta_{\text {sup }}^{3}\right]\|\eta\|_{1, \Omega}^{2}+\frac{1}{P e}\left[4 \varepsilon_{1} \delta_{1} \theta_{\text {inf }}^{3}+B i\right]\|\eta\|_{0, \Gamma_{f}}^{2} \\
& \geq\left[\frac{1}{P e\left(C_{\Omega}^{2}+1\right)}-\frac{4}{P e} \varepsilon_{1} \delta_{1} \theta_{\text {sup }}^{3}\right]\|\eta\|_{1, \Omega}^{2}
\end{aligned}
$$

Finally, we deduce from the expression of $\delta_{1}\left(\delta_{1}=\frac{L^{*} \sigma\left(\theta^{*}\right)^{3}}{\lambda}\right)$ that if $\lambda>\lambda_{\text {min }}$ then the bilinear form $l(u ; .,$.$) is V_{0}$-elliptic and the result follows from the Lax-Milgram theorem.

Remark 2 Let us consider the case meas $\left(\Gamma_{n}\right)=0$. Hence $\partial \Omega=\Gamma_{d} \cup \Gamma_{f}$ and for all $\eta \in V_{0}(\Omega),\|\eta\|_{0, \partial \Omega}=\|\eta\|_{0, \Gamma_{f}}$. Therefore, (3.29) becomes:

$l(u ; \eta, \eta) \geq \frac{1}{P e\left(C_{\Omega}^{2}+1\right)}\|\eta\|_{1, \Omega}^{2}-\frac{1}{P e}\left[4 \delta_{1}\left(\varepsilon_{1} \theta_{\text {sup }}^{3}-\varepsilon_{0} \theta_{i n f}^{3}\right)-B i\right]\|\eta\|_{0, \partial \Omega}^{2}$

and the upper bound we obtain in order to have the problem $\left(P^{l}\right)$ well posed is slightly different. It is the following:

either

$$
h \geq 4 \sigma\left(\theta^{*}\right)^{3} \varepsilon_{0} \theta_{i n f}^{3}\left[\frac{\varepsilon_{1} \theta_{\text {sup }}^{3}}{\varepsilon_{0} \theta_{\text {inf }}^{3}}-1\right]
$$


or

$$
\left\{\begin{array}{l}
h<4 \sigma\left(\theta^{*}\right)^{3} \varepsilon_{0} \theta_{\text {inf }}^{3}\left[\frac{\varepsilon_{1} \theta_{\text {sup }}^{3}}{\varepsilon_{0} \theta_{\text {inf }}^{3}}-1\right] \\
\text { and } \\
\lambda>L^{*}\left(C_{\Omega}^{2}+1\right)\left[4 \sigma\left(\theta^{*}\right)^{3} \varepsilon_{0} \theta_{\text {inf }}^{3}\left(\frac{\varepsilon_{1} \theta_{\text {sup }}^{3}}{\varepsilon_{0} \theta_{\text {inf }}^{3}}-1\right)-h\right]
\end{array}\right.
$$

The condition (3.30) does not depend on $\lambda$. It expresses that if the convective exchanges on the boundary are sufficiently large compared to the radiative ones then the linearized problem is well posed. In other respects, when $h=0$ the condition (3.31) is less restrictive than (3.25).

Remark 3 Let us recall that the formulation of the problem considered in ${ }^{14}$ is different. This formulation presents the advantage to take into account the case of black bodies i.e. $\varepsilon=1$; and, it is proved in ${ }^{9}$, Lemma 6 , that the linearized problem is coercitive in $H^{1}(\Omega)$ (hence well posed) under a condition very similar to (3.30).

Uniqueness of the solution Following an idea of $\left({ }^{14}\right.$, Theorem 2$)$, we obtain straightforwardly the uniqueness of the physical solution of $\left(P^{w}\right)$ when the linearized problem is well posed.

Proposition 5 Under the assumptions of Theorem 1 and if the thermal conductivity $\lambda$ is strictly greater than $\lambda_{\min }$ then there exists a unique solution $(\theta, w) \in$ $H^{1}(\Omega) \times L^{\infty}(\partial \Omega)$ to Problem $\left(P^{w}\right)$ which satisfies (3.16) (i.e. a unique physical solution).

Proof. Following the proof of existence of ${ }^{11}$, we prove that the truncated problem $\left(P^{\bar{Q}}\right)$ has a minimal solution (in $H^{1}(\Omega)$ ) denoted by $\theta_{-}$and a maximal solution (in $H^{1}(\Omega)$ ) denoted by $\theta^{+}$. Now, let us prove that when the linearized problem is well posed (i.e. when $\lambda>\lambda_{\min }$ ), this minimal solution $\theta_{-}$and this maximal solution $\theta^{+}$ of $\left(P^{\bar{Q}}\right)$ are equals. By this end, we write $\eta=\left(\theta^{+}-\theta_{-}\right)$and we have:

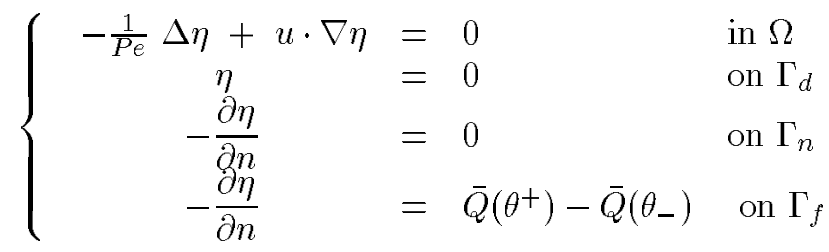

In other respects, there exists a function $\tilde{\theta} \in L^{\infty}(\partial \Omega), \theta_{\text {inf }} \leq \tilde{\theta}(x) \leq \theta_{\text {sup }}$ a.e. such that: $\left(\theta^{+}\right)^{4}-\left(\theta_{-}\right)^{4}=4 \tilde{\theta}^{3}\left(\theta^{+}-\theta_{-}\right)=4 \tilde{\theta}^{3} \eta$. Therefore,

$-\frac{\partial \eta}{\partial n}(x)=B i \eta(x)+4 \delta_{1}\left[\varepsilon(x) \tilde{\theta}^{3}(x) \eta(x)-\frac{\varepsilon}{1-\varepsilon}(x) \int_{\partial \Omega} K(x, y) \varepsilon(y) \tilde{\theta}^{3}(y) \eta(y) d s(y)\right]$

It follows from Proposition 4 that if $\lambda>\lambda_{\min }$, then the truncated problem $\left(P^{\bar{Q}}\right)$ has a unique solution $\theta$ in $H^{1}(\Omega)$ and this solution satisfies (3.16). Therefore, the problem $\left(P^{Q}\right)$ has a unique solution $\theta$ in $H^{1}(\Omega)$ which satisfies (3.16). Using Proposition 1 , we obtain the result. 


\section{Numerical Analysis}

\subsection{Introduction and Setting of the Result}

In this section, we write a numerical analysis related to a truncated problem. For a technical reason, this truncated problem is slightly different from the previous one, however, under the assumptions of Proposition 5, it has a unique solution which is the unique physical solution of $\left(P^{w}\right)$. We assume that $\Omega$ is a polyhedric domain of $\mathbb{R}^{n}$ ( $n=2$ or 3$)$ and using a standard finite element method, we discretize the equations. The main result of this section is Theorem 2: under the assumption that the linearized problem is well posed (here when $\lambda>\lambda_{\min }$ ), we prove the existence and uniqueness of the discrete solution and its convergence towards the unique physical solution of the initial problem when the mesh size $h$ is small enough. (We write the numerical analysis of the centered scheme i.e we do not consider a stabilization procedure). In order to obtain these results, we use the framework of 1 . Notice that this framework does not require the discrete maximum principle.

A new truncated problem Let us define a new truncated problem:

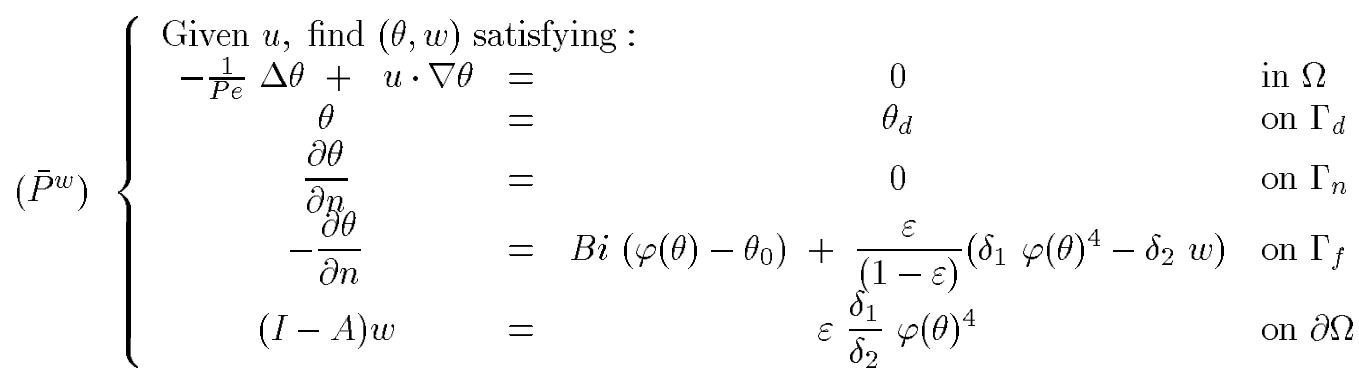

where $A$ is the operator defined by (3.5) and $\varphi$ is the $C^{2}$-truncation defined in what follows. Let $\xi$ be a real strictly positive such that $\theta_{\text {inf }}-\xi>0$. We define:

$$
\varphi(t)=\left\{\begin{array}{clc}
\theta_{\text {inf }} & \text { if } & t \leq \theta_{\text {inf }}-\xi \\
P_{\text {inf }}(t) & \text { if } & \theta_{\text {inf }}-\xi \leq t \leq \theta_{\text {inf }} \\
t & \text { if } & \theta_{\text {inf }} \leq t \leq \theta_{\text {sup }} \\
P_{\text {sup }}(t) & \text { if } & \theta_{\text {sup }} \leq t \leq \theta_{\text {sup }}+\xi \\
\theta_{\text {sup }} & \text { if } & \theta_{\text {sup }}+\xi \leq t(x)
\end{array}\right.
$$

where $P_{\text {inf }}(t)$ and $P_{\text {sup }}(t)$ are polynomials such that $\varphi(t)$ defined from $\mathbb{R}$ into itself is of class $C^{2}$. Namely, $P_{\text {inf }}$ is such that:

$$
\left\{\begin{array}{c}
P_{\text {inf } f}\left(\theta_{\text {inf }}-\xi\right)=P_{\text {inf }}\left(\theta_{\text {inf }}\right)=\theta_{\text {inf }} \\
P_{\text {inf }}^{\prime}\left(\theta_{\text {inf }}-\xi\right)=0 ; P_{\text {inf }}^{\prime}\left(\theta_{\text {inf }}\right)=1 \\
P_{\text {inf }}^{\prime \prime}\left(\theta_{\text {inf }}-\xi\right)=P_{\text {inf }}^{\prime \prime}\left(\theta_{\text {inf }}\right)=0
\end{array}\right.
$$

The condition (4.33) defines a unique polynomial $P_{\text {inf }}$ of degree 5 . Similarly, there exists a unique polynomial $P_{\text {sup }}$ of degree 5 which fulfills the conditions such that $\varphi(t)$ is $C^{2}$.

Let us make some remarks concerning the introduction of this new truncated problem. Firstly, we introduce a truncated problem because of the lack of regularity of the boundary condition in the $3 \mathrm{~d}$ case. As a matter of fact, in order to write the 
numerical analysis, we formulate the problem as a fixed point problem and this lack of regularity on $\Gamma_{f}$ prevents us from doing it in the standard Sobolev space $H^{1}(\Omega)$. In other respects, we do not consider the same truncated problem as in the continuous analysis because the technique of the numerical analysis which follows, requires a $C^{2}$ truncation.

Secondly, we consider a truncated problem in order to discretize a well posed problem. As a matter of fact, we proved in Section 3 that when $\lambda$ is large enough, the initial problem $\left(P^{w}\right)$ has a unique physical solution but we did not prove the non existence of eventual non physical solutions i.e. solutions which do not satisfy the weak maximum principle (3.16). On the other hand, the truncated problem $\left(\bar{P}^{w}\right)$ has a unique solution in $H^{1}(\Omega)$ and this solution satisfies the weak maximum principle (see below).

Assumption 3 The temperatures $\theta_{d}$ and $\theta_{0}$ given on the boundary are such that:

$$
\inf _{\Gamma_{d}} \theta_{d}<\inf _{\Gamma_{f}} \theta_{0}, \sup _{\Gamma_{f}} \theta_{0}<\sup _{\Gamma_{d}} \theta_{d}
$$

and the parameter of truncation $\xi$ is small enough.

Under Assumption 3, we have the existence and uniqueness of the solution to this new truncated problem $\left(\bar{P}^{w}\right)$. As a matter of fact, let $\bar{Q}_{\varphi}(\theta)$ be the operator analogue to the operator $\bar{Q}(\theta)$ defined by (3.13), but relative to the $C^{2}$-truncation $\varphi$ :

$$
\begin{aligned}
\bar{Q}_{\varphi}(\theta)(x)= & B i\left(\varphi(\theta)-\theta_{0}\right)(x)+\left[\varepsilon(x) \delta_{1} \varphi(\theta)^{4}(x)\right. \\
& \left.-\frac{\varepsilon(x)}{1-\varepsilon(x)} \int_{\partial \Omega} K(x, y) \varepsilon(y) \delta_{1} \varphi(\theta)^{4}(y) d s(y)\right]
\end{aligned}
$$

We can easily check that when Assumption 3 holds, the operator $\bar{Q}_{\varphi}$ satisfies the properties of Lemma 1 (with the constants $K$ and $M$ depending on $\xi$ ). The result of existence stated in Theorem 1 and the result of uniqueness (Proposition 5) remain true. Therefore, under the assumptions of Proposition 5 and when Assumption 3 holds, there exists a unique solution $(\theta, w) \in H^{1}(\Omega) \times L^{\infty}(\partial \Omega)$ to the new truncated problem $\left(\bar{P}^{w}\right)$ and this solution satisfies the weak maximum principle (3.16). Therefore, this solution is the unique physical solution of the initial problem $\left(P^{w}\right)$.

The discrete spaces We assume that $\Omega$ is a polyhedric domain of $\mathbb{R}^{n}$ ( $n=2$ or 3) and we discretize Problem $\left(\bar{P}^{w}\right)$ with the help of a classical finite element method. We denote by $\left(\mathcal{T}_{h}\right)$ a regular and quasi-uniform family of triangulation, $\bar{\Omega}=\cup_{\left(T \in \mathcal{T}_{h}\right)} T$. We associate this family of triangulation to a single reference Lagrange finite element of class $\mathcal{C}^{0}$ (see e.g. ${ }^{4}$ ).

Let $k$ be an integer greater or equal than 1 . We define:

$$
U_{h}(\Omega)=\left\{t \in \mathcal{C}^{0}(\bar{\Omega}) ; \forall T \in \mathcal{T}_{h},\left.t\right|_{T} \in P_{k}\right\}
$$

and

$$
V_{0 h}(\Omega)=\left\{t \in U_{h} ;\left.t\right|_{\Gamma_{d}}=0\right\}
$$

We suppose that $\theta_{d}$ is the trace of a function of $U_{h}$ and we define:

$$
V_{t h}(\Omega)=\left\{t \in U_{h} ;\left.t\right|_{\Gamma_{d}}=\theta_{d}\right\}
$$


We define:

$$
\mathcal{W}_{h}(\partial \Omega)=\mid \begin{array}{ll}
\{v: \partial \Omega \rightarrow \mathbb{R}, v \text { piecewise constant }\} & \text { if } k=1 \\
\left\{v \in \mathcal{C}^{0}(\partial \Omega) ; \text { for every } T \in \mathcal{T}_{h}\right. \text { such that } & \\
\text { a boundary of } \left.\partial T \in \partial \Omega,\left.v\right|_{\partial T} \in P_{k-1}\right\} & \text { if } k \geq 2
\end{array}
$$

The spaces $V_{0 h}(\Omega)$ and $V_{t h}(\Omega)$ are both subsets of $H^{1}(\Omega)$ and $\mathcal{W}_{h}(\partial \Omega)$ is a subset of $L^{2}(\partial \Omega)$.

The discrete problem We denote by $u_{h}$ a finite element approximation of the fluid velocity $u$. The discrete formulation of $\left(\bar{P}^{w}\right)$ is the following:

$$
\left(\bar{P}_{h}^{w}\right)\left\{\begin{array}{l}
\text { Given } u_{h}, \text { find }\left(\theta_{h}, w_{h}\right) \in V_{t h}(\Omega) \times \mathcal{W}_{h}(\partial \Omega) \text { such that: } \\
\forall t_{h} \in V_{0 h}(\Omega), a\left(u_{h} ; \theta_{h}, t_{h}\right) \\
\quad+\frac{1}{P e} \int_{\Gamma_{f}}\left[\operatorname{Bi}\left(\varphi\left(\theta_{h}\right)-\theta_{0}\right)+\frac{\varepsilon}{(1-\varepsilon)}\left(\delta_{1} \varphi\left(\theta_{h}\right)^{4}-\delta_{2} w_{h}\right)\right] t_{h} d s=0 \\
\forall v_{h} \in \mathcal{W}_{h}(\partial \Omega), \\
\int_{\partial \Omega}(I-A) w_{h} v_{h} d s=\frac{\delta_{1}}{\delta_{2}} \int_{\partial \Omega} \varepsilon \varphi\left(\theta_{h}\right)^{4} v_{h} d s
\end{array}\right.
$$

where $a(. ; .,$.$) is the trilinear mapping defined by (3.18).$

An intermediate problem Let us introduce the following linear decoupled problem:

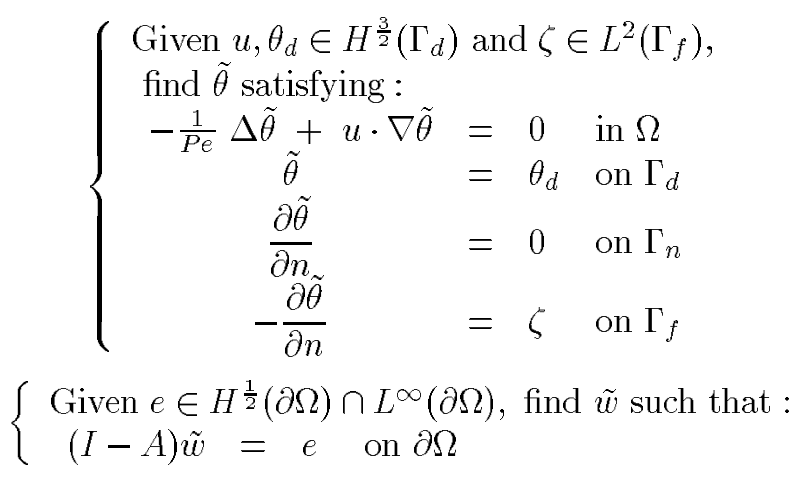

It follows from the Lax-Milgram theorem and Proposition 1 that under the assumptions 1 and 2 , this linear decoupled system (4.38)-(4.39) has a unique solution $(\tilde{\theta}, \tilde{w})$ in $H^{1}(\Omega) \times L^{\infty}(\partial \Omega)$. Later, we will be required to write the numerical analysis of this problem (4.38)-(4.39). Hence, let us specify the weak formulations and the corresponding discrete formulations. The weak formulation of (4.38) is:

$$
\left\{\begin{array}{l}
\text { Given } u \text { and } \zeta \in L^{2}\left(\Gamma_{f}\right), \text { find } \tilde{\theta} \in V_{t}(\Omega) \text { such that: } \\
\forall t \in V_{0}(\Omega), a(u ; \tilde{\theta}, t)=\int_{\Gamma_{f}} \zeta t d s
\end{array}\right.
$$

We write a weak formulation of (4.39) in $L^{2}(\partial \Omega)$ :

$$
\left\{\begin{array}{l}
\text { Given } e \in H^{\frac{1}{2}}(\partial \Omega) \cap L^{\infty}(\partial \Omega), \text { find } \tilde{w} \in L^{2}(\partial \Omega) \text { such that: } \\
\forall v \in L^{2}(\partial \Omega), \mathcal{R}(\tilde{w}, v)=\int_{\partial \Omega} e v d s
\end{array}\right.
$$


where $\mathcal{R}$ is the bilinear form defined from $L^{2}(\partial \Omega) \times L^{2}(\partial \Omega)$ into $\mathbb{R}$ by:

$$
\mathcal{R}(w, v)=\int_{\partial \Omega}(I-A) w v d s
$$

The discrete formulations are the following:

$$
\begin{gathered}
\left\{\begin{array}{l}
\text { Given } u_{h} \text { and } \zeta \in L^{2}\left(\Gamma_{f}\right) \text {, find } \tilde{\theta}_{h} \in V_{t h}(\Omega) \text { such that: } \\
\forall t_{h} \in V_{0 h}(\Omega), a\left(u_{h} ; \tilde{\theta}_{h}, t_{h}\right)=\int_{\Gamma_{f}} \zeta t_{h} d s
\end{array}\right. \\
\left\{\begin{array}{l}
\text { Given } e \in H^{\frac{1}{2}}(\partial \Omega) \cap L^{\infty}(\partial \Omega), \text { find } \tilde{w}_{h} \in \mathcal{W}_{h}(\partial \Omega) \text { such that: } \\
\forall v_{h} \in \mathcal{W}_{h}(\partial \Omega), \mathcal{R}\left(\tilde{w}_{h}, v_{h}\right)=\int_{\partial \Omega} e v_{h} d s
\end{array}\right.
\end{gathered}
$$

We make the following assumptions.

Assumption 4 (Regularity and convergence of the velocity fluid). There exists a real $\alpha, \alpha \geq \frac{1}{2}$, such that $u \in H^{\alpha}(\Omega)$ and:

$$
\left\|u-u_{h}\right\|_{0, \partial \Omega}=O\left(h^{\alpha}\right)
$$

Assumption 5 (Regularity of $(\tilde{\theta}, \tilde{w})$ ). There exist real $\beta$ and $\gamma$ strictly greater than $\left(\frac{n}{2}-1\right)$ such that $(\tilde{\theta}, \tilde{w})$, the unique solution to (4.38)-(4.39), belongs to $H^{1+\beta}(\Omega) \times$ $H^{\gamma}(\partial \Omega)$.

The main result of this section is the following:

Theorem 2 Let the assumptions 1, 2, 3, 4 and 5 be satisfied and let the emissivity $\varepsilon$ be a lipschitz function. If the thermal conductivity $\lambda$ is strictly greater than $\lambda_{\text {min }}$ and if the mesh size $h$ is small enough, then there exists a unique solution to the discrete problem $\left(\bar{P}_{h}^{w}\right)$.

In addition, for all $\varepsilon^{\prime}>0$, there exists a constant $C_{\varepsilon^{\prime}}$ independent of $h$ such that

$$
\left\|\theta-\theta_{h}\right\|_{1, \Omega}+\left\|w-w_{h}\right\|_{0, \partial \Omega} \leq C_{\varepsilon^{\prime}} h^{\alpha_{\varepsilon^{\prime}}}
$$

where $\alpha_{\varepsilon^{\prime}}=\operatorname{Min}\left(\alpha-\varepsilon^{\prime}, 1-\frac{n}{2}+\beta-\varepsilon^{\prime}, \gamma\right)$ (n being the space dimension) and $(\theta, w)$ is the unique solution of $\left(\bar{P}^{w}\right)$. (In others words, the unique discrete solution of $\left(\bar{P}_{h}^{w}\right)$ converge to the unique physical solution of the initial problem $\left.\left(P^{w}\right)\right)$.

In the remainder of the paper, we suppose that the assumptions 1, 2, 3, 4 and 5 are satisfied.

In order to prove Theorem 2, we use the analysis written by Brezzi, Rappaz and Raviart ${ }^{1}$ dealing with finite dimensional approximation of nonlinear problems of the form $F(\lambda ; x)=0$, where $F: \Lambda \times X \rightarrow X$ for some interval $\Lambda \subset \mathbb{R}$ and some Banach space $X$. We consider branches of non-singular solutions to the problem (i.e. a solution $x(\lambda)$ such that $D_{x} F(\lambda ; x)$ is an isomorphism of $\left.X\right)$ and we make use of an extended implicit function theorem in order to obtain existence and uniqueness of the discrete solution and its convergence. This analysis is notably applied to the study of the convergence of some finite element methods for Navier-Stokes equations $\left(\sec ^{1}, 6\right)$.

The proof of Theorem 2 is done in four steps which follow.

4.2. First step. The Continuous and Discrete Problems Formulated as Fixed Point Problems

We formulate problems $\left(\bar{P}^{w}\right)$ and $\left(\bar{P}_{h}^{w}\right)$ as fixed point problems. 
Continuous problem We define:

$$
X=H^{1}(\Omega) \times L^{2}(\partial \Omega) \text { and } Y=L^{2}\left(\Gamma_{f}\right) \times H^{\frac{1}{2}}(\partial \Omega) \cap L^{\infty}(\partial \Omega) \times H^{\frac{3}{2}}\left(\Gamma_{d}\right)
$$

and let $\Lambda$ be a compact interval of $\mathbb{R}^{*+}$ included into $] \lambda_{\min },+\infty\left[\right.$ (the bound $\lambda_{\min }$ is defined by (3.25).

We define the linear operator $T$ as follows:

$$
T: Y \rightarrow X ;\left(\zeta, e, \theta_{d}\right) \mapsto-(\tilde{\theta}, \tilde{w})
$$

where $\tilde{x}=(\tilde{\theta}, \tilde{w}) \in X$ is the solution of the linear decoupled system (4.38)-(4.39). Let $\lambda$ be the thermal conductivity, we define the non-linear operator $G$ by:

$$
G(\lambda ; t, v)=\left(B i\left(\varphi(t)-\theta_{0}\right)+\frac{\varepsilon}{(1-\varepsilon)}\left(\delta_{1} \varphi(t)^{4}-\delta_{2} v\right), \varepsilon \frac{\delta_{1}}{\delta_{2}} \varphi(t)^{4}, \theta_{d}\right)
$$

where $\varphi$ is the $C^{2}$-truncation defined by (4.32). (Let us recall that the operators above depend on $\lambda$ via the dimensionless numbers $P e, B i, \delta_{1}$ and $\delta_{2}$ ).

Let us remark that for all $t \in H^{1}(\Omega), \varphi(t)^{4} \in H^{\frac{1}{2}}(\partial \Omega) \cap L^{\infty}(\partial \Omega)$. Furthermore, if the emissivity $\varepsilon$ is regular enough (for example a lipschitz function) then

$$
\varepsilon \frac{\delta_{1}}{\delta_{2}} \varphi(t)^{4} \in H^{\frac{1}{2}}(\partial \Omega) \cap L^{\infty}(\partial \Omega)
$$

The temperature $\theta_{0}$ is a positive function given in $H^{\frac{1}{2}}\left(\Gamma_{f}\right) \cap L^{\infty}\left(\Gamma_{f}\right)$, then for all $(t, v) \in H^{1}(\Omega) \times L^{2}(\partial \Omega)$, the term

$$
B i\left(\varphi(t)-\theta_{0}\right)+\frac{\varepsilon}{(1-\varepsilon)}\left(\delta_{1} \varphi(t)^{4}-\delta_{2} v\right)
$$

belongs to $L^{2}\left(\Gamma_{f}\right)$ and $G$ is a $C^{2}$ operator from $\Lambda \times X$ into $Y$.

Remark 4 If in the definition of $G$ we do not truncate the right-hand side of the integral equation, then we only have

$$
\varepsilon \frac{\delta_{1}}{\delta_{2}} t^{4} \in L^{1}(\partial \Omega)
$$

and Assumption 5 cannot be satisfied.

We define $F$ from $\Lambda \times X$ into $X$ by:

$$
F(\lambda, x)=x+T G(\lambda, x) \quad \forall \lambda \in \Lambda, \forall x \in X
$$

Then, Problem $\left(\bar{P}^{w}\right)$ is equivalent to the following problem:

$$
\left(P^{F}\right)\left\{\begin{array}{c}
\text { Given } \lambda \in \Lambda, \text { find } x=(\theta, w) \in X \text { such that : } \\
F(\lambda ; x)=0
\end{array}\right.
$$


Discrete problem We define the finite dimensional space

$$
X_{h}=V_{t h}(\Omega) \times \mathcal{W}_{h}(\partial \Omega)
$$

We have $X_{h} \subset X$. We define the operator $T_{h}$ as follows:

$$
T_{h}: Y \rightarrow X_{h} ;\left(\zeta, e, \theta_{d}\right) \mapsto-\left(\tilde{\theta}_{h}, \tilde{w}_{h}\right)
$$

where $\tilde{x}_{h}=\left(\tilde{\theta}_{h}, \tilde{w}_{h}\right) \in X_{h}$ is solution of (4.42)-(4.43) and we define:

$$
F_{h}\left(\lambda ; x_{h}\right)=x_{h}+T_{h} G\left(\lambda ; x_{h}\right)
$$

The discrete problem $\left(\bar{P}_{h}^{w}\right)$ is equivalent to the following problem:

$$
\left(P_{h}^{F}\right)\left\{\begin{array}{c}
\text { Given } \lambda \in \Lambda, \text { find } x_{h}=\left(\theta_{h}, w_{h}\right) \in X_{h} \text { such that : } \\
F_{h}\left(\lambda ; x_{h}\right)=0
\end{array}\right.
$$

Let us recall the theorem we will use later. This theorem is due to F. Brezzi, J. Rappaz and P.A. Raviart, ${ }^{1}$ (see also the work of M. Crouzeix and J. Rappaz, ${ }^{5}$ ). Theorem $3(1,5)$. If the following assumptions are satisfied:

i) the operator $G$ is $C^{p}$ (with $p \geq 2$ ) from $\Lambda \times X$ into $Y$ and $D_{x}^{p} G$ is bounded in any bounded sub-space of $\Lambda \times X$,

ii) there exists $Z$ a Banach space included into $Y$ with continuous injection, such that: $D_{x} G(\lambda, x) \in \mathcal{L}(X, Z) \quad \forall \lambda \in \Lambda, \forall x \in X$

iii)

$$
\lim _{h \rightarrow 0}\left\|\left(T-T_{h}\right) y\right\|_{X}=0 \quad \forall y \in Y
$$

and

$$
\lim _{h \rightarrow 0}\left\|\left(T-T_{h}\right)\right\|_{\mathcal{L}(Z, X)}=0
$$

iv) and $\{(\lambda, x(\lambda)) ; \lambda \in \Lambda\}$ is a branch of non-singular solutions of Problem $\left(P^{F}\right)$.

Then, there exists a neighborhood $\mathcal{V}$ of the origin in $X$ and for a mesh size $h$ small enough, a unique function $\lambda \in \Lambda \mapsto x_{h}(\lambda) \in X_{h}$ of class $C^{p}$ such that $\left\{\left(\lambda, x_{h}(\lambda)\right) ; \lambda \in \Lambda\right\}$ is a branch of non singular solutions to Problem $\left(P_{h}^{F}\right)$ and $\left(x(\lambda)-x_{h}(\lambda)\right) \in \mathcal{V}$ for all $\lambda \in \Lambda$.

In addition, there exists a constant $C$ independent of $h$ and $\lambda$ such that :

$$
\left\|x(\lambda)-x_{h}(\lambda)\right\|_{X} \leq C\left\|\left(T-T_{h}\right)(G(\lambda, x(\lambda)))\right\|_{X} \quad \forall \lambda \in \Lambda
$$

We prove in the next steps that the assumptions of Theorem 3 are satisfied for problems $\left(P^{F}\right)$ and $\left(P_{h}^{F}\right)$.

\subsection{Second Step. Linearized Problem}

Let $\lambda$ be given and $x(\lambda)$ be the unique solution of $\left(P^{F}\right)$. We state a sufficient condition on $\lambda$ such that $\{(\lambda, x(\lambda))\}$ is a branch of non singular solutions i.e. such that $D_{x} F(\lambda ; x)$ is an isomorphism of $X$.

Lemma 2 Let $\lambda_{\min }$ be the real defined by (3.25). If the thermal conductivity $\lambda$ is strictly greater than $\lambda_{\min }$ then $D_{x} F(\lambda ; x)$ is an isomorphism of $X$. 
Proof. The linear operator $D_{x} F(\lambda ; x)$ is an isomorphism of $X$ if and only if for all $y=(\xi, v) \in X$, there exists a unique $z=(\eta, r) \in X$ such that

$$
z+T\left(D_{x} G(\lambda ; x) \cdot z\right)=y
$$

and if there exists a constant $c>0$ such that: $\|z\|_{X} \leq c|| y \|_{X}$.

Let us recall that since $(\theta, w)$ is the unique solution of $\left(P^{F}\right), \theta$ satisfies the weak maximum principle (3.16) and $\varphi(\theta)=\theta$ a.e. on $\partial \Omega$. Hence, Equation (4.53) is equivalent to:

$$
\begin{aligned}
& -\frac{1}{P e} \Delta \eta+u \cdot \nabla \eta=\quad-\frac{1}{P e} \Delta \xi+u \cdot \nabla \xi \quad \text { in } \Omega \\
& \eta=\xi \quad \text { on } \Gamma_{d} \\
& \frac{\partial \eta}{\partial n}=\quad \frac{\partial \xi}{\partial n} \quad \text { on } \Gamma_{n} \\
& -\frac{\partial \eta}{\partial n}=-\frac{\partial \xi}{\partial n}+B i \eta+\frac{\varepsilon}{(1-\varepsilon)}\left(4 \delta_{1} \theta^{3} \eta-\delta_{2} r\right) \quad \text { on } \Gamma_{f} \\
& (I-A) r=\quad(I-A) v+4 \varepsilon \frac{\delta_{1}}{\delta_{2}} \theta^{3} \eta \quad \text { on } \partial \Omega
\end{aligned}
$$

It follows from Proposition 1 that $D_{x} F(\lambda ; x)$ is an isomorphism of $X$ if and only if for all $(\xi, v) \in X$, there exists a unique $\eta \in H^{1}(\Omega)$ which satisfies:

$$
\begin{aligned}
& -\frac{1}{P e} \Delta \eta+u \cdot \nabla \eta=-\frac{1}{P e} \Delta \xi+u \cdot \nabla \xi \quad \text { in } \Omega \\
& \eta=\quad \xi \quad \text { on } \Gamma_{d} \\
& \frac{\partial \eta}{\partial n}=\quad \frac{\partial \xi}{\partial n} \quad \text { on } \Gamma_{n} \\
& -\frac{\partial \eta}{\partial n}=\quad \phi(\xi, v ; \eta) \quad \text { on } \Gamma_{f}
\end{aligned}
$$

where

$$
\begin{aligned}
\phi(\xi, v ; \eta)(x)= & L(\eta)(x)-\frac{\partial \xi}{\partial n}(x)-\delta_{2} \frac{\varepsilon}{1-\varepsilon}(x)[(I-A) v(x) \\
& \left.+\int_{\partial \Omega} K(x, y)(I-A) v(y) d s(y)\right] \quad \text { a.e. on } \partial \Omega
\end{aligned}
$$

with $L(\eta)$ defined by (3.23). Finally, the result follows from Proposition 4.

\subsection{Third Step. Error Estimates on the Linear Decoupled System}

In this section, we prove that the relation (4.50) holds. To this end, we prove that the operator $T_{h}$ defined by (4.48) is well defined from $Y$ into $X_{h}$ (propositions 6 and 8), we write an estimate of the finite element error $\left\|\left(T-T_{h}\right)(y)\right\|_{X}$ for all $y \in Y$ (propositions 7 and 8 ), and finally (4.50) follows.

\subsubsection{The Linear Partial Differential Equation}

Proposition 6 If the mesh size $h$ is small enough, Problem (4.42) has a unique solution. 
Proof. We prove that the bilinear mapping $a\left(u_{h} ;, .,\right)$ defined by $(3.18)$ is $V_{0 h^{-}}$ elliptic when the mesh size $h$ is small enough. Notice that the relation $\operatorname{div}\left(u_{h}\right)=0$ is a priori not satisfied as it is in the continuous case. We write:

$$
a\left(u_{h} ; \theta_{h}, \theta_{h}\right)=a\left(u ; \theta_{h}, \theta_{h}\right)-\int_{\Omega}\left(u-u_{h}\right) \nabla\left(\frac{\theta_{h}^{2}}{2}\right) d x
$$

Using the relation (3.22) and Hölder's inequality, we deduce that there exist positives constants $C_{1}$ and $C_{2}$ such that:

$$
\begin{aligned}
a\left(u_{h} ; \theta_{h}, \theta_{h}\right) & \geq C_{1}\left\|\theta_{h}\right\|_{1, \Omega}^{2}-C_{2}\left\|u-u_{h}\right\|_{0, \Omega}\left\|\nabla\left(\frac{\theta_{h}^{2}}{2}\right)\right\|_{0, \Omega} \\
& \geq C_{1}\left\|\theta_{h}\right\|_{1, \Omega}^{2}-C_{2}\left\|u-u_{h}\right\|_{0, \Omega}\left\|\theta_{h}\right\|_{1, \Omega}^{2}
\end{aligned}
$$

and using Assumption 4, we deduce there exists a constant $C_{u}$ independent of $h$ such that:

$$
a\left(u_{h} ; \theta_{h}, \theta_{h}\right) \geq\left(C_{1}-C_{u} h^{\alpha}\right)\left\|\theta_{h}\right\|_{1, \Omega}^{2}
$$

Therefore, the mapping $a\left(u_{h} ; . ..\right)$ is $V_{0 h}$-elliptic for $h$ sufficiently small and the result follows from Lax-Milgram's theorem.

Let $\tilde{\theta}$ and $\tilde{\theta}_{h}$ be respectively the solutions of (4.40) and (4.42), we establish an error estimate of $\left\|\tilde{\theta}-\tilde{\theta}_{h}\right\|$ under norm $H^{1}$. The coupling term $\int_{\Omega} u \nabla \tilde{\theta} t d x$ in the equation (4.40) prevents us from applying Céa's lemma (see e.g. ${ }^{1}$ ). Nevertheless, we adapt the same idea of proof.

Lemma 3 Let $\tilde{\theta}$ and $\tilde{\theta}_{h}$ be respectively the solutions of (4.40) and (4.42). For all $t_{h} \in V_{0 h}(\Omega)$, we have:

$$
a\left(u ; \tilde{\theta}-\tilde{\theta}_{h}, t_{h}\right)+\int_{\Omega}\left(u-u_{h}\right) \nabla \tilde{\theta}_{h} t_{h} d x=0
$$

Proof. We subtract the equation (4.40) from the equation (4.42) with $t=t_{h}$ as a test function $\left(t_{h} \in V_{0 h}(\Omega) \subset V_{0}(\Omega)\right)$. We obtain:

$$
\forall t_{h} \in V_{0 h}(\Omega), a\left(u ; \tilde{\theta}, t_{h}\right)-a\left(u_{h} ; \tilde{\theta}_{h}, t_{h}\right)=0
$$

hence,

$$
a\left(u ; \tilde{\theta}-\tilde{\theta}_{h}, t_{h}\right)+a\left(u ; \tilde{\theta}_{h}, t_{h}\right)-a\left(u_{h} ; \tilde{\theta}_{h}, t_{h}\right)=0
$$

which gives (4.63).

We denote by $\Pi_{h}^{k}$ the Lagrange interpolation operator from $H^{s}(\Omega), s>\frac{n}{2}$, into $V_{0 h}(\Omega)$ of degree $k$ (let us recall that functions belonging to $H^{s}(\Omega), s>\frac{n}{2}$ are continuous in $\bar{\Omega})$. Further we denote by $P_{h}$ the operator of projection from $V_{0}(\Omega)$ onto $V_{0 h}(\Omega)$ in the sense of the semi-norm $H_{0}^{1}$ i.e. $P_{h} v$ is defined by:

$$
\int_{\Omega} \nabla\left(v-P_{h} v\right) \nabla v_{h} d x=0 \quad \forall v_{h} \in V_{0 h}(\Omega)
$$

We recall 
Lemma 4 Let $s$ and $t$ be two reals such that: $s>\frac{n}{2}, 1 \leq t \leq s$. Then for all $v \in H^{s}(\Omega) \cap V_{0}(\Omega)$, there exists a constant $C$ independent of $h$ such that:

i)

$$
\left\|v-P_{h} v\right\|_{t, \Omega} \leq C h^{s-t}\|v\|_{s, \Omega}
$$

ii) For all $\varepsilon, 0<\varepsilon<s-\frac{n}{2}$,

$$
\left\|v-P_{h} v\right\|_{0, \infty, \Omega} \leq C h^{s-\frac{n}{2}-\varepsilon}\|v\|_{s, \Omega}
$$

The proof of this lemma is done in ${ }^{8}$, Theorem 3.2 and Corollary 3.3 .

We deduce the following

Lemma 5 Let $v$ be a function in $V_{0}(\Omega) \cap H^{s}(\Omega)$ with $s>\frac{n}{2}$. There exists a constant $C$ independent of $h$ such that for all $v_{h} \in V_{0 h}(\Omega)$, for all $\varepsilon>0$ and for all $q>n$,

$$
\left\|v-v_{h}\right\|_{0, \infty, \Omega} \leq C\left(h^{s-\frac{n}{2}-\varepsilon}\|v\|_{s, \Omega}+h^{\frac{n}{q}-\frac{n}{2}}\left\|v-v_{h}\right\|_{1, \Omega}\right)
$$

Proof. We write:

$$
\forall v_{h} \in V_{0 h}(\Omega),\left\|v-v_{h}\right\|_{0, \infty, \Omega} \leq\left\|v-P_{h} v\right\|_{0, \infty, \Omega}+\left\|v_{h}-P_{h} v\right\|_{0, \infty, \Omega}
$$

Let us bound by above the term $\left\|v_{h}-P_{h} v\right\|_{0, \infty, \Omega}$. The injection from $W^{1, q}(\Omega)$ into $L^{\infty}(\Omega)$ is continuous for all $q>n$, hence:

$$
\left\|v_{h}-P_{h} v\right\|_{0, \infty, \Omega} \leq C\left\|v_{h}-P_{h} v\right\|_{1, q, \Omega} \text { for all } q>n
$$

We apply an inverse inequality to $\left(v_{h}-P_{h} v\right) \in V_{0 h}(\Omega)$ (see e.g. ${ }^{4}$, Theorem 17.2):

$$
\left|v_{h}-P_{h} v\right|_{1, q, \Omega} \leq C h^{\frac{n}{q}-\frac{n}{2}}\left|v_{h}-P_{h} v\right|_{1, \Omega}
$$

Then, we deduce from Poincaré's inequality that:

$$
\begin{aligned}
\left\|v_{h}-P_{h} v\right\|_{0, \infty, \Omega} & \leq C h^{\frac{n}{q}-\frac{n}{2}}\left\|v_{h}-P_{h} v\right\|_{1, \Omega} \\
& \leq C h^{\frac{n}{q}-\frac{n}{2}}\left(\left\|v-v_{h}\right\|_{1, \Omega}+\left\|v-P_{h} v\right\|_{1, \Omega}\right)
\end{aligned}
$$

and we deduce from (4.65) (with $t=1$ ) that:

$$
\left\|v_{h}-P_{h} v\right\|_{0, \infty, \Omega} \leq C\left(h^{\frac{n}{q}-\frac{n}{2}}\left\|v-v_{h}\right\|_{1, \Omega}+h^{s-1+\frac{n}{q}-\frac{n}{2}}\|v\|_{s, \Omega}\right)
$$

Finally, we obtain the result by combining (4.66)(4.67) and (4.68).

Proposition 7 Let $\tilde{\theta}$ and $\tilde{\theta}_{h}$ be the solutions of (4.40) and (4.42). Under assumptions 4 and 5 and for all $\varepsilon>0$, there exists a positive constant $C_{\varepsilon}$ independent of $h$ such that:

$$
\left\|\tilde{\theta}-\tilde{\theta}_{h}\right\|_{1, \Omega} \leq C_{\varepsilon} h^{\tilde{\alpha}-\varepsilon}\|\tilde{\theta}\|_{1+\beta, \Omega}
$$

where $\tilde{\alpha}=\min \left(\alpha, 1-\frac{n}{2}+\beta\right)$.

Proof. The mapping $a(u ;, .$,$) is V_{0}$-elliptic, hence there exists $\alpha>0$ such that:

$$
a\left(u ; \tilde{\theta}-\tilde{\theta}_{h}, \tilde{\theta}-\tilde{\theta}_{h}\right) \geq \alpha\left\|\tilde{\theta}-\tilde{\theta}_{h}\right\|_{1, \Omega}^{2}
$$


And the equation (4.63) gives: $\forall t_{h} \in V_{0 h}(\Omega)$,

$$
a\left(u ; \tilde{\theta}-\tilde{\theta}_{h}, \tilde{\theta}-\tilde{\theta}_{h}\right)=a\left(u ; \tilde{\theta}-\tilde{\theta}_{h}, \tilde{\theta}-t_{h}\right)-\int_{\Omega}\left(u-u_{h}\right) \nabla \tilde{\theta}_{h}\left(t_{h}-\tilde{\theta}_{h}\right) d x
$$

Using Hölder's inequality, we find an upper bound for the first term of (4.71):

$$
\left|a\left(u ; \tilde{\theta}-\tilde{\theta}_{h}, \tilde{\theta}-t_{h}\right)\right| \leq C\left\|\tilde{\theta}-\tilde{\theta}_{h}\right\|_{1, \Omega}\left[\left\|\tilde{\theta}-t_{h}\right\|_{1, \Omega}+\|u\|_{0, \Omega}\left\|\tilde{\theta}-t_{h}\right\|_{0, \infty, \Omega}\right]
$$

We then find an upper bound for the second term of (4.71):

$$
\begin{aligned}
\left|\int_{\Omega}\left(u-u_{h}\right) \nabla \tilde{\theta}_{h}\left(t_{h}-\tilde{\theta}_{h}\right) d x\right| \leq & \left\|u-u_{h}\right\|_{0, \Omega}\left[\left\|\tilde{\theta}-\tilde{\theta}_{h}\right\|_{1, \Omega}+\|\tilde{\theta}\|_{1, \Omega}\right] \\
& \times\left[\left\|\tilde{\theta}-\tilde{\theta}_{h}\right\|_{0, \infty, \Omega}+\left\|\tilde{\theta}-t_{h}\right\|_{0, \infty, \Omega}\right]
\end{aligned}
$$

We write $\tau_{h}=\left\|\tilde{\theta}-\tilde{\theta}_{h}\right\|_{1, \Omega}$ and combining (4.70), (4.71), (4.72) and (4.73), we obtain:

$$
\begin{aligned}
\tau_{h}^{2} & \leq C \tau_{h}\left[\left\|\tilde{\theta}-t_{h}\right\|_{1, \Omega}+\|u\|_{0, \Omega}\left\|\tilde{\theta}-t_{h}\right\|_{0, \infty, \Omega}\right] \\
& +C\left\|u-u_{h}\right\|_{0, \Omega}\left[\tau_{h}+\|\tilde{\theta}\|_{1, \Omega}\right]\left[\left\|\tilde{\theta}-\tilde{\theta}_{h}\right\|_{0, \infty, \Omega}+\left\|\tilde{\theta}-t_{h}\right\|_{0, \infty, \Omega}\right]
\end{aligned}
$$

for all $t_{h} \in V_{0 h}(\Omega)$. We write $t_{h}=P_{h} \tilde{\theta}, P_{h}$ being defined by (4.64). Then, it follows from assumptions 4 and 5 , lemmas 4 and 5 that:

$$
\begin{array}{r}
{\left[1-O\left(h^{n / q-n / 2+\alpha}\right)\right] \tau_{h}^{2}-\left[O\left(h^{1+\beta-n / 2-\varepsilon}\right)+O\left(h^{n / q-n / 2+\alpha}\right)\right] \tau_{h}} \\
-O\left(h^{1+\beta-n / 2-\varepsilon+\alpha}\right) \leq 0
\end{array}
$$

where $\varepsilon>0$ and $q$ is any real such that $q>n$. Hence, let us choose $q$ such that $\frac{n}{2}-\frac{n}{q}=\varepsilon$. We write a Taylor's expansion of $\left(1-O\left(h^{n / q-n / 2+\alpha}\right)\right)$ in the neighborhood of 0 and we obtain the following second order inequation:

$$
\tau_{h}^{2}-\left[O\left(h^{1+\beta-n / 2-\varepsilon}\right)+O\left(h^{\alpha-\varepsilon}\right)\right] \tau_{h}-O\left(h^{1+\beta-n / 2+\alpha-\varepsilon}\right) \leq 0
$$

Let $\tau_{1}$ and $\tau_{2}$ be the solutions to the corresponding equation. They are real and $\tau_{h}$ is such that $\tau_{1} \leq \tau_{h} \leq \tau_{2}$. In addition, $\tau_{1} \tau_{2}<0$ hence $\tau_{1}<0$. Then, we find:

$$
\tau_{h}=\left\|\tilde{\theta}-\tilde{\theta}_{h}\right\|_{1, \Omega} \leq \tau_{2}=O\left(h^{\tilde{\alpha}-\varepsilon}\right)
$$

with $\tilde{\alpha}=\min \left(\alpha, 1-\frac{n}{2}+\beta\right)$, and the proof is complete.

\subsubsection{The Integral Equation}

Proposition 8 There exists unique solutions $\tilde{w}$ and $\tilde{w}_{h}$ respectively to problems (4.41) and (4.43). In addition, under Assumption 5, there exists a positive constant $C$ independent of $h$ such that:

$$
\left\|\tilde{w}-\tilde{w}_{h}\right\|_{0, \partial \Omega} \leq C h^{\gamma}\|\tilde{w}\|_{\gamma, \partial \Omega}
$$


Proof. Let us prove that the bilinear mapping $\mathcal{R}$ is elliptic in $L^{2}(\partial \Omega)$. We have: $\forall w \in L^{2}(\partial \Omega), \mathcal{R}(w, w)=\int_{\partial \Omega}(I-A) w^{2} d s$ and $\int_{\partial \Omega} A w^{2} d s \leq\|A\|_{0, \infty}\|w\|_{0, \partial \Omega}^{2}$. It is proved in ${ }^{10}$ that $A$ is a contracting operator from $L^{\infty}(\partial \Omega)$ into $L^{\infty}(\partial \Omega)($ i.e. $\left.\|A\|_{0, \infty}<1\right)$, hence:

$$
\begin{aligned}
\mathcal{R}(w, w) & \geq\left(1-\|A\|_{0, \infty}\right)\|w\|_{0, \partial \Omega}^{2} \\
& \geq C\|w\|_{0, \partial \Omega}^{2} \quad \text { with } C>0
\end{aligned}
$$

It follows from Lax-Milgram's theorem that problems (4.41) and (4.43) have respectively a unique solution. Further, it follows from the $L^{2}$-ellipticity of $\mathcal{R}(.,$.$) and its$ continuity from $L^{2}(\partial \Omega) \times L^{2}(\partial \Omega)$ into $\mathbb{R}$ that:

$$
\left\|\tilde{w}-\tilde{w}_{h}\right\|_{0, \Omega} \leq C\left\|\tilde{w}-v_{h}\right\|_{0, \Omega} \quad \forall v_{h} \in V_{0}(\Omega)
$$

Let $s_{h}$ be the $L^{2}$-local projection operator from $L^{2}(\partial \Omega)$ into $P_{k-1}, k$ integer $\geq 1$, defined as follows: $s_{h} v \in P_{k-1}, \int_{\partial T}\left(s_{h} v-v\right) w_{h} d s=0$ for all $w_{h} \in P_{k-1}$ and for all $\partial T \in \partial \Omega, T \in \mathcal{T}_{h}$. Let $\gamma$ be a real, $0<\gamma \leq k$, for all $v \in H^{\gamma}(\partial \Omega)$ there exists a positive constant $C$ independent of $h$ such that (see e.g. ${ }^{6}$ p102):

$$
\left\|v-s_{h} v\right\|_{0, \partial \Omega} \leq C h^{\gamma}|v|_{\gamma, \partial \Omega}
$$

Then, the error estimate (4.75) follows from Assumption 5, (4.76) and (4.77).

\subsection{Fourth Step. Conclusion and Results}

Let us recall that the assumptions 1, 2, 3, 4 and 5 hold. Then, we verify that the four assumptions i)-iv) of Theorem 3 are satisfied.

Using the regularity of the truncation operator $\varphi$, the operator $G$ defined by (4.46) is $C^{2}$ from $\Lambda \times X$ into $Y$. And, $D_{x}^{2} G(\lambda ; x)$ is a bounded operator in any bounded sub-space of $\Lambda \times X$. We choose $Z=Y$ and we obtain $D_{x} G(\lambda ; x) \in \mathcal{L}(X ; Z) \quad \forall \lambda \in$ $\Lambda, \forall x \in X$. Hence, assumptions $i$ ) and ii) are satisfied.

Assumption iv) follows from Lemma 2.

Let $\tilde{\theta}$ and $\tilde{\theta}_{h}$ be the solutions respectively to problems (4.40) and (4.42) and let $\tilde{w}$ and $\tilde{w}_{h}$ be the solutions respectively to problems (4.41) and (4.43). It follows from Proposition 7 and Proposition 8 that for all $\varepsilon>0$ and under assumptions 4 and 5 , there exists a constant $C_{\varepsilon}$ independent of $h, \tilde{\theta}$ and $\tilde{w}$ such that:

$$
\begin{aligned}
\forall y \in Z=Y,\left\|\left(T-T_{h}\right)(y)\right\|_{X} & =\left\|\left(\tilde{\theta}-\tilde{\theta}_{h}\right)(h)\right\|_{1, \Omega}+\left\|\left(\tilde{w}-\tilde{w}_{h}\right)(g)\right\|_{0, \partial \Omega} \\
& \leq C_{\varepsilon} h^{\alpha_{\varepsilon}}\left(\|\tilde{\theta}\|_{1+\beta, \Omega}+\|\tilde{w}\|_{\gamma, \partial \Omega}\right)
\end{aligned}
$$

where $\alpha_{\varepsilon}=\operatorname{Min}\left(\alpha-\varepsilon, 1-\frac{n}{2}+\beta-\varepsilon, \gamma\right)$. Therefore relations (4.50) and (4.51) are satisfied.

Finally, we apply Theorem 3. The estimate (4.52) gives:

$$
\begin{aligned}
\left\|x(\lambda)-x_{h}(\lambda)\right\|_{X} & \leq C\left\|\left(T-T_{h}\right)(G(\lambda, x(\lambda)))\right\|_{X} \\
& \leq C_{\varepsilon} h^{\alpha_{\varepsilon}}\left(\|\tilde{\theta}\|_{1+\beta, \Omega}+\|\tilde{w}\|_{\gamma, \partial \Omega}\right)
\end{aligned}
$$

which states (4.44) and Theorem 2. 


\section{Linear Systems}

In this section, we consider the discrete problem $\left(\bar{P}_{h}^{w}\right)$ with the truncation $\varphi \equiv I d$ i.e we consider the discretization of the initial problem $\left(P^{w}\right)$. We present linear systems and algorithms we use in order to solve this problem.

\subsection{The Integral Equation}

Let the discrete temperature $\theta_{h}$ be given on the boundary $\partial \Omega$, we compute the discrete radiosity $w_{h}$, the solution of the integral equation.

We denote by $\left\{X_{i}\right\}_{1<i<r}$ characteristic functions of surfaces $(\partial \Omega)^{i} .\left\{X_{i}\right\}_{1 \leq i \leq r}$ is a base of $\mathcal{W}_{h}(\partial \Omega)$. We seek $w_{h}$ in the space $\mathcal{W}_{h}(\partial \Omega)$ defined as follows:

$w_{h}(x)=\sum_{i=1}^{r} w_{h}^{i} X_{i}(x)$. We assume that the emittance $\varepsilon$ is constant on surfaces. Therefore, it belongs to $\mathcal{W}_{h}(\partial \Omega)$. We write:

. $S_{i j}=S_{i} F_{i j}=\int_{(\partial \Omega)^{i}} \int_{(\partial \Omega)^{j}} \phi(x, y) d s(y) d s(x)$.

where $S_{i}$ is the surface of $(\partial \Omega)^{i}$ and $F=\left(F_{i j}\right), 1 \leq i, j \leq r$ is the angle factor matrix (see e.g. ${ }^{12}$ ). The computation of this matrix is done using a Monte-Carlo method and shadow effects are taken into account. More details concerning its computation can be found in ${ }^{7}$.

. $e_{h}^{i}=\frac{\delta_{1}}{\delta_{2}} \int_{(\partial \Omega)^{i}} \varepsilon \theta_{h}^{4} d s$

. $S=\left(S_{i j}\right), 1 \leq i, j \leq r ; \quad E_{h}=\left(e_{h}^{i}\right)$ and $W_{h}=\left(w_{h}^{i}\right), \quad 1 \leq i \leq r$.

- $D_{\varepsilon}=\operatorname{diag}\left(\varepsilon_{i}\right), 1 \leq i \leq r$

. and $M=\operatorname{diag}\left(S_{i}\right)-\left[I-D_{\varepsilon}\right] S$. We have:

$$
M_{i j}=\mid \begin{array}{lll}
S_{i} & \text { if } \quad i=j \\
-\left(1-\varepsilon_{i}\right) S_{i j} & \text { if } \quad i \neq j
\end{array}
$$

The matrix $S$ is symmetric and the matrix $M$ is positive definite. The linear system we solve is :

$$
\left\{\begin{array}{l}
\text { Given } E_{h}, \text { find } W_{h} \in \mathbb{R}^{r} \text { such that: } \\
M W_{h}=E_{h}
\end{array}\right.
$$

\subsection{The Partial Differential Equation}

Let $w_{h}$ be given on $\partial \Omega$. We compute $\theta_{h}$, the solution of the non linear partial differential equation. More precisely, we solve the following problem:

$$
\left\{\begin{array}{l}
\text { Given } u_{h} \text { and } w_{h}, \text { find } \theta_{h} \in V_{t h}(\Omega) \text { such that: } \\
\forall t_{h} \in V_{0 h}(\Omega), \quad a\left(u_{h} ; \theta_{h}, t_{h}\right) \\
+\frac{1}{P e} \int_{\Gamma_{f}}\left[B i\left(\theta_{h}-\theta_{0}\right)+\frac{\varepsilon}{(1-\varepsilon)}\left(\delta_{1} \theta_{h}^{4}-\delta_{2} w_{h}\right)\right] t_{h} d s=0
\end{array}\right.
$$

We use a Newton-Raphson's algorithm to solve $(5.80)$. We denote by $\left(V_{0 h}(\Omega)\right)^{\prime}$ the dual space of $V_{0 h}(\Omega)$ and we define the operator $A_{h}^{w}$ as follows:

$$
A_{h}^{w}: V_{t h}(\Omega) \rightarrow\left(V_{0 h}(\Omega)\right)^{\prime}: \theta_{h} \mapsto A_{h}^{w}\left(\theta_{h}\right)
$$


where

$$
\begin{aligned}
\left\langle A_{h}^{w}\left(\theta_{h}\right), t_{h}\right\rangle= & a\left(u_{h} ; \theta_{h}, t_{h}\right) \\
& \quad+\frac{1}{P e} \int_{\Gamma_{f}}\left[B i\left(\theta_{h}-\theta_{0}\right)+\frac{\varepsilon}{(1-\varepsilon)}\left(\delta_{1} \theta_{h}^{4}-\delta_{2} w_{h}\right)\right] t_{h} d s \\
= & 0
\end{aligned}
$$

$<,>$ is the scalar product in the duality $\left(V_{0 h}(\Omega)\right)^{\prime}, V_{0 h}(\Omega)$. It is obvious that the operator $A_{h}^{w}$ is differentiable from $V_{t h}(\Omega)$ into $\left(V_{0 h}(\Omega)\right)^{\prime}$. At iterate $n$, we solve the following linear system:

$$
\left\{\begin{array}{l}
\text { Given } u_{h}, w_{h} \text { and } \theta_{h}^{n}, \text { find } \theta_{h}^{n+1} \in V_{t h}(\Omega) \text { such that: } \forall t_{h} \in V_{0 h}(\Omega), \\
<\left(A_{h}^{w}\right)^{\prime}\left(\theta_{h}^{n}\right) \cdot \theta_{h}^{n+1}, t_{h}>=<\left(A_{h}^{w}\right)^{\prime}\left(\theta_{h}^{n}\right) \cdot \theta_{h}^{n}, t_{h}>-<A_{h}^{w}\left(\theta_{h}^{n}\right), t_{h}>
\end{array}\right.
$$

We denote by $\left(t_{i}\right)_{i=1, m}$ a base of $V_{0 h}(\Omega)$ and we seek $\theta_{h}^{n+1}$ in the form:

$\theta_{h}^{n+1}(x)=\sum_{i=1}^{m}\left(\theta_{h}^{n+1}\right)^{i} t_{i}(x)$ ( $m$ is the number of nodes). We denote:

$$
\begin{aligned}
& \Theta_{h}^{n}=\left(\left(\theta_{h}^{n}\right)^{i}\right)_{i=1, m} \\
& A_{i j}=a\left(u_{h} ; t_{i}, t_{j}\right)+\frac{1}{P e} \int_{\Gamma_{f}}\left[B i+4 \frac{\varepsilon}{(1-\varepsilon)} \delta_{1}\left(\theta_{h}^{n}\right)^{3}\right] t_{i} t_{j} d s \\
& B_{j}\left(\Theta_{h}^{n}, W_{h}\right)=\frac{1}{P e} \int_{\Gamma_{f}}\left[B i \theta_{0}+\frac{\varepsilon}{(1-\varepsilon)}\left(3 \delta_{1}\left(\theta_{h}^{n}\right)^{4}+\delta_{2} w_{h}\right)\right] t_{j} d s
\end{aligned}
$$

The linear problem (5.81) becomes:

$$
\left\{\begin{array}{l}
\text { Given } W_{h} \text { and } \Theta_{h}^{n}, \text { find } \Theta_{h}^{n+1} \in \mathbb{R}^{m} \text { such that: } \\
A \cdot \Theta_{h}^{n+1}=B\left(\Theta_{h}^{n}, W_{h}\right)
\end{array}\right.
$$

The matrix $A$ is sparse, non-symmetric and positive definite.

In order to avoid numerical instabilities, we introduce artificial diffusion into the numerical scheme in a classical manner. We use a streamline diffusion method and the expression of $a\left(u_{h} ; .,.\right)$ is modified as follows:

$$
\begin{aligned}
\tilde{a}\left(u_{h} ; t_{i}, t_{j}\right) & =a\left(u_{h} ; t_{i}, t_{j}\right)+\frac{1}{P e} \int_{\Omega} \tau_{h} \nabla t_{i} \nabla t_{j} d x \\
& =\frac{1}{P e} \int_{\Omega}\left(I+\tau_{h}\right) \nabla t_{i} \nabla t_{j} d x+\int_{\Omega} u_{h} \nabla t_{i} t_{j} d x
\end{aligned}
$$

where $\tau_{h}$ is the matrix of artificial diffusion. It is positive definite and its terms vanish with $h$. Its expression is: $\tau_{h}=\frac{h}{2}(P e)^{2} u_{h}{ }^{T} u_{h}$.

\subsection{Algorithm of Resolution}

In order to solve Problem $\left(P_{h}^{w}\right)$, we use the following relaxation algorithm:

a. Initialization: $\Theta_{h}^{0}$ given.

b. Let $\Theta_{h}^{n}$ known on $\partial \Omega$, compute $W_{h}^{n}$ the solution of (5.79).

c. Let $W_{h}^{n}$ and $\Theta_{h}^{n}$, compute $\Theta_{h}^{n+1}$ the solution of (5.82). 
d. Test: if $\frac{\left\|\Theta_{h}^{n+1}-\Theta_{h}^{n}\right\|}{\left\|\Theta_{h}^{n}\right\|}<\varepsilon$ then stop. If not, re-iterate.

In the numerical tests we performed, we computed $\Theta_{h}^{0}$ as the solution of the thermal model without the radiative terms.

We do not study the convergence of this algorithm. Nevertheless, we never had any problem in practice concerning its convergence.

\section{Numerical Results}

We consider a twodimensional air flow under a car bonnet. Parts under the bonnet are modeled as indicated on Figure 1. Physical data corresponds to a car moving at 80 kilometers per hour (Peclet's number Pe equals $10^{5}$ ).

The fluid flow is a stationary incompressible potential flow. The normal velocity of the flow is known on the air entrance (i.e. on the radiator exit) and equals $5 \mathrm{~m} / \mathrm{s}$. The fluid potential is known on exit. The condition $u . n$ equals 0 is imposed at all others places on the boundary.

Temperatures are imposed (Dirichlet's condition) on the air entrance $\left(280^{\circ} \mathrm{K}\right)$, on the engine block $\left(420^{\circ} \mathrm{K}\right)$ and on the exhaust pipe $\left(520^{\circ} \mathrm{K}\right)$. The thermal flow $\nabla \theta . n$ equals 0 on the air exit and we consider at all others places (i.e. on the external surface, on the hose, on the screen and on the battery) heat transfer described by relation (2.4). The emittance $\varepsilon$ depends on $x$ and is such that $0.5<\varepsilon<0.8$ everywhere on the boundary except on the air exit. As a matter of fact, from a radiative point of view, we model the air exit as a black body $(\varepsilon=1)$ at a given temperature $\theta_{\text {exit }}=320^{\circ} \mathrm{K}$.

The software we have used has been written by A. Habbal, working for the companies SIMULOG S.A. and RENAULT S.A. and then, has been completed by the first author of this paper in order to incorporate the radiation effects. This software is written into the library Modulef (INRIA/SIMULOG). We make a piecewise constant approximation for the radiosity $w$ and a piecewise linear approximation for the temperature $\theta$. The angle factors $\phi(x, y)$ are computed by a software written by A. Mezrhab using a Monte-Carlo's method, ${ }^{7}$. Shadow effects are taken into account.

\section{Conclusion}

We wrote a mathematical analysis and a numerical analysis of the thermal model which takes into account the following types of heat transfer: convection, diffusion and radiation of grey bodies separated by a non participating media. The initial motivation of this study was a shape optimal design problem as applied to the automotive industry, ${ }^{2}, 3$.

In order to prove the present analysis, we used a technique of truncation which consists of truncating the non linear, non monotone and non local boundary condition to "natural" values i.e. to the inf-sup values of the temperature given on the boundary. Then, we proved: i) the existence and uniqueness of the physical solution in the standard Sobolev space $H^{1}(\Omega)$; and ii) the existence, uniqueness and convergence of a finite element solution. These results hold under the condition that the linearized problem (Problem $\left(P^{l}\right)$, Section 3.2) is well posed in $H^{1}(\Omega)$. We further proved that this linearized problem is well posed when the thermal conductivity $\lambda$ is large enough compared to the radiative data.

In other respects, we would like to point out that our present analysis can be easily extended to the weakly coupled model Navier-Stokes equations / present thermal equations. As a matter of fact, we assumed that the fluid flow is incompressible and $u$ belongs to $\left(L^{p}(\Omega)\right)^{n}$ with $p>n$. Therefore if $u$ is a solution of the steady state Navier-Stokes equations, the velocity field satisfies these assumptions. In addition, 


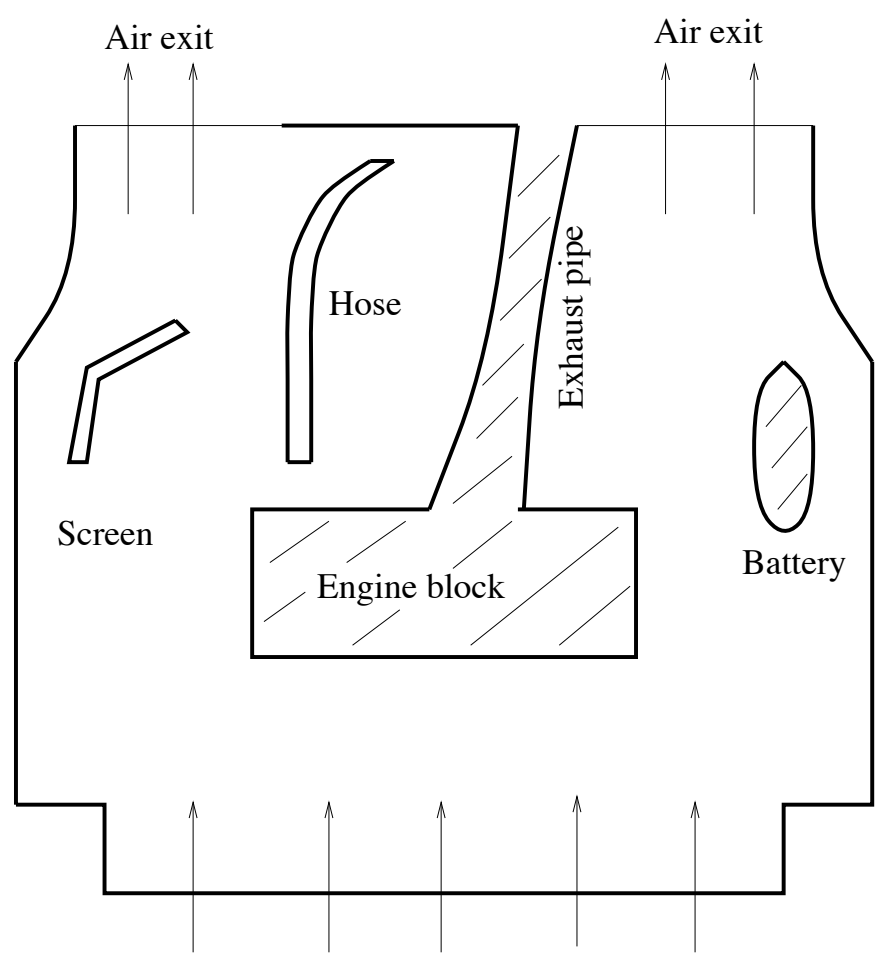

Air entrance

Figure 1: Engine scheme 


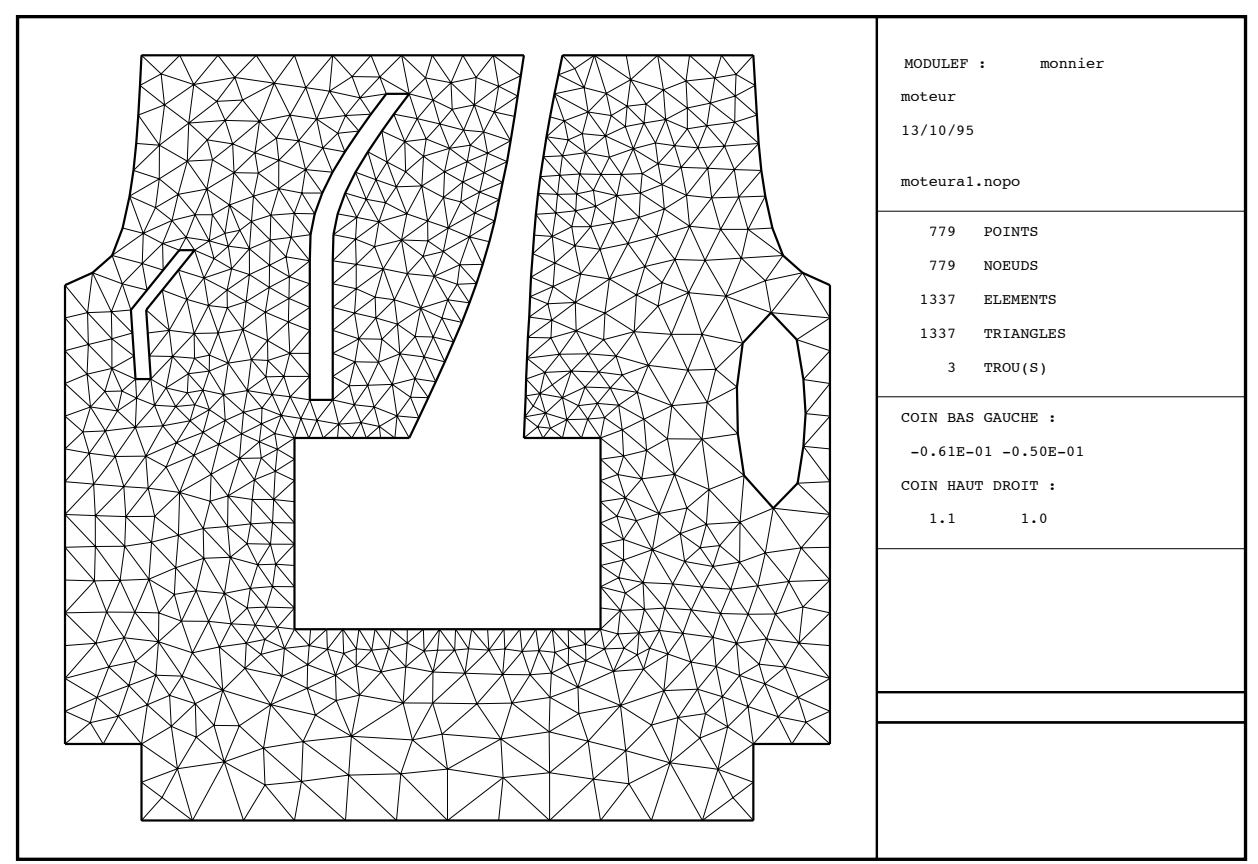

Figure 2: Mesh

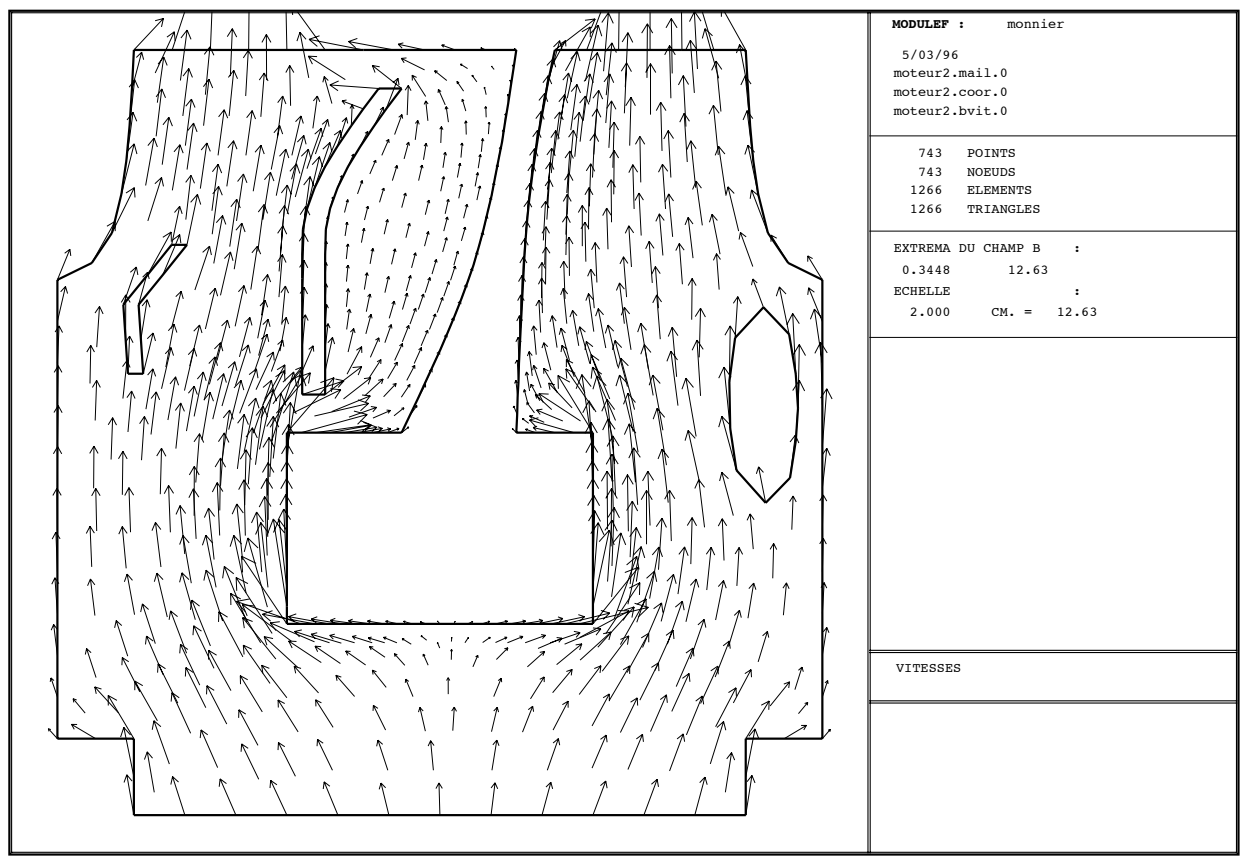

Figure 3: Fluid velocity (incompressible potential flow) 


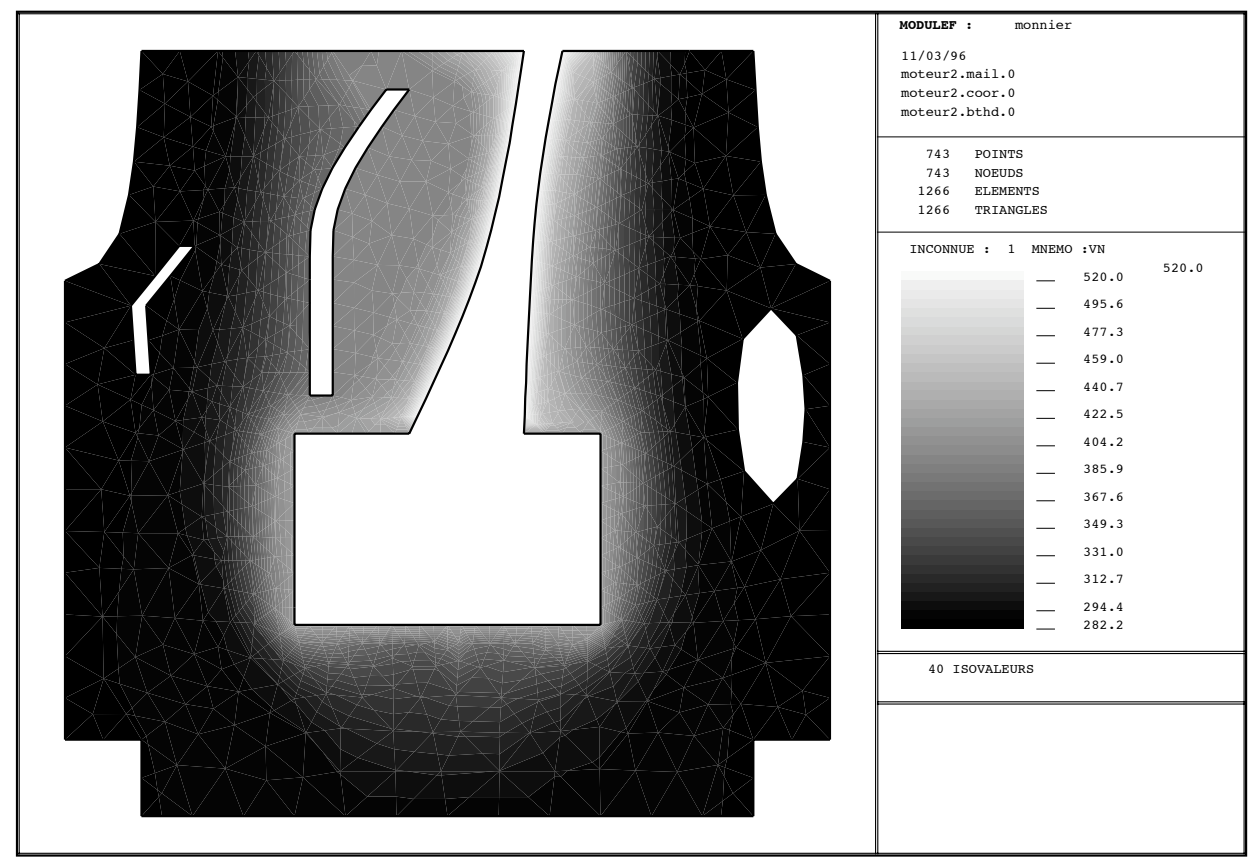

Figure 4: Isotherms

it is proved in ${ }^{1}$ and ${ }^{6}$ that the Navier-Stokes equations considered in the stream function formulation or in the velocity-pressure formulation fit into the framework of ${ }^{1}$. Let $\nu$ denotes the fluid viscosity coefficient, if $\nu$ is small enough (or equivalently if the Reynolds number is small enough) then the linearized Navier-Stokes equations are well posed, ${ }^{6}$. Under this condition, the Navier-Stokes equations have a branch of non singular solutions.

In other respects, the convergence of mixed finite element methods (in two dimensional space) using the stream function formulation and conforming finite element methods using the velocity-pressure formulation can be proved using Theorem 3, 1,6 . Therefore, from these results and the analysis presented in this paper, we can directly obtain, if the Reynolds is small enough and if the thermal conductivity $\lambda$ is sufficiently large, the existence and uniqueness of a branch of non singular solutions to the (weakly) coupled system of steady state Navier-Stokes equations and to the thermal problem $\left(P^{w}\right)$. In addition, if we use an adapted finite element method, the existence, uniqueness and convergence of the corresponding $C^{2}$ branch of discrete solutions are obtained.

\section{Acknowledgment}

This work and the software development were partially supported by the companies Renault S.A. and Simulog S.A. The authors gratefully acknowlege them. Also, the authors would like to thank the referee for his helpful comments. 


\section{References}

1. F. Brezzi, J. Rappaz and P.A. Raviart, Finite-dimensionnal approximation of non-linear problems. Part I: branches of nonsingular solutions, Num. Math. 36 (1980) 1-25.

2. D. Chenais, J. Monnier and J.P. Vila, Shape optimal design for a fluid-heat coupled system, Appl. Math. and Comp. Sc., vol. 6, No2, (1996) 245-261.

3. D. Chenais, J. Monnier and J.P. Vila, A shape optimal design problem with convective and radiative heat transfer. Analysis and implementation., J. Optim. Th. Appl., To appear.

4. P.G. Ciarlet, Basic error estimates for elliptic problems, in Handbook of numerical analysis. Vol. II. Finite element methods (part 1), eds P.G. Ciarlet and J.L. Lions (North-Holland, Amsterdam, 1991).

5. M. Crouzeix and J. Rappaz, On numerical approximation in bifurcation theory, (Masson, RMA, 1990).

6. V. Girault and P.A. Raviart, Finite element method for Navier-Stokes equations, (Springer-Verlag, Paris, 1986).

7. A. Mezrhab, Contribution à l'étude numérique et expérimentale des échanges radiatifs, Thesis, University of Provence, 1991.

8. J. Monnier, Conception optimale de forme pour un système couplé fluide-thermique. Application à l'aérothermique d'un véhicule, Thesis, University of Nice-Sophia Antipolis (1995).

9. J. Monnier, Free convection with radiative thermal transfer of grey bodies. Analysis and approximation by finite element methods, Math. Models Meth. Appl. Sc., 9 (10) 2000.

10. C. Perret, Simulation numérique des échanges thermiques et des contraintes thermoélastiques dans un tirage Czochralski, Thesis, University of J. Fourier - Grenoble I (1989).

11. C. Perret and P. Witomski, Équation de la chaleur et réflections multiples, Annales Institut Poincaré, 8(6) (1991) 677-689.

12. E.M. Sparrow and R.D. Cess, Radiation heat transfer, (Mc Graw-Hill Book Co, New-York, 1978).

13. T. Tiihonen, Stefan-Boltzmann radiation on non-convex surfaces, Math. Meth. in Appl. Sc., 20 (1997) 47-57.

14. T. Tiihonen, A nonlocal problem arising from heat radiation on non-convex surfaces, Euro. Jnl of Appl. Math., 8 (1997) 403-416.

15. T. Tïhonen, Finite element approximation of nonlocal heat radiation problems, Math. Models Meth. Appl. Sc., 8(6) (1998) 1071-1089. 